\title{
Computational modeling of human papillomavirus with impulsive vaccination
}

\author{
Hailay Weldegiorgis Berhe ${ }^{\circ}$ \\ Mo'tassem Al-arydah $\mathbb{D}$
}

Received: 4 August 2020 / Accepted: 29 November 2020 / Published online: 8 January 2021

(C) The Author(s), under exclusive licence to Springer Nature B.V. part of Springer Nature 2021

\begin{abstract}
In this study, a new SIVS epidemic model for human papillomavirus (HPV) is proposed. The global dynamics of the proposed model are analyzed under pulse vaccination for the susceptible unvaccinated females and males. The threshold value for the disease-free periodic solution is obtained using the comparison theory for ordinary differential equations. It is demonstrated that the disease-free periodic solution is globally stable if the reproduction number is less than unity under some defined parameters. Moreover, we found the critical value of the pulse vaccination for susceptible females needed to control the HPV. The uniform persistence of the disease for some parameter values is also analyzed. The numerical simulations conducted agreed with the theoretical findings. It is found out using numerical simulation that the pulse vaccination has a good impact on reducing the disease.
\end{abstract}

Keyword Human papillomavirus · Impulsive vaccination - Global attractivity - Uniform persistence

\footnotetext{
H. W. Berhe $(\varangle)$

Department of Mathematics, Mekelle University, Mekelle, Ethiopia

e-mail: hailaywg@yahoo.com

M. Al-arydah

Department of Mathematics, Khalifa University, Abu Dabi, UAE e-mail: motassem.alarydah@ku.ac.ae
}

\section{Introduction}

Human papillomavirus (HPV) remains a major public health problem and is continuous receiving global attention [1]. It has been known as the primary etiologic agent of cervical, anal, penile, vaginal, vulvar, and head/neck cancers; anogenital warts; and recurrent respiratory papillomatoses [2]. There exist more than 100 different forms of human papillomavirus. An estimate of 30 to 40 strains or types are sexually transmitted; disease transmission depends on age and sexual behavior. Such diseases affect the human genital tract. The most common causes of these genital warts and laryngeal papillomatoses are HPV6 and HPV11. Previous studies indicate that cervical cancer is the most important cause of death for women. According to [3], around 70\% of cervical cancers are caused by HPV16 and HPV 18. Currently, vaccination against HPV for both men and women is considered to be the most important tool to halt the disease from wide-spreading globally.

Several authors have used mathematical modeling to investigate the dynamics of infectious diseases using continuous vaccination and have found vaccination an efficient implementation in reducing the spread of the disease [4-7]. We know, however, that continuous vaccination is not realistic in reality living, and the control steps are only employed in the specific moment. So for this reason, disease models with the implementation of impulsive vaccination are studied by more and more scholars. Pulse vaccination strategy (PVS) 
consists of periodical repetitions of impulsive vaccines for the targeted population. During each vaccination period, a constant fraction, $p$ of susceptible people are vaccinated. This kind of vaccination is called impulsive because all the vaccine doses are administered in a time which is very short with respect to the nature of the target disease [8-12].

Relatively rich findings have been obtained for the theories of impulsive differential equations [13]. Impulsive differential equations are differential equations with impulse effects that act like a normal reflection of the observed evolution of many real-world problems. Initially, impulsive differential equations were developed to help in the modeling of physical phenomena involving impulsive physical problems, biological and ecological systems, industrial robotics, pharmacokinetics, biotechnology, and optimal control theory [14]. Some scholars, for instance, the authors [8,1517], have studied the basic SIR and SIS models as impulsive differential equations by applying pulse vaccination. Currently, there are also published reports of the dynamic study of the infectious disease models with pulse vaccination [12,18-23]. For example, the authors [19] investigated the global stability of a SIR epidemic model with impulsive vaccination, and the authors [18] investigated an optimal control impulsive SQEIAR epidemic of COVID-19; the authors [12] also analyzed a SIR optimal impulse vaccination model for a short period of time.

For the past decade, researchers have published on the analysis of the epidemiological modeling of HPV. Most of the previous scholars have focused on the homogeneous deterministic models [24-28]. In addition to this, the age-structured deterministic assumption is becoming more evident since most sexually transmitted diseases are age-dependent. To that end, researches on HPV transmission dynamics assumed the age-dependent disease transmission dynamics [2931]. But to our knowledge, the HPV model with pulse vaccination is not studied by previous scholars, and therefore, the impulsive systems with application to HPV are new to the literature. We also believe that the related stability problems are fascinating and challenging. The most important challenge is handling the system of equations. Impulsive differential equations incorporate both the discrete and continuous systems. We get systems of difference equations by characterizing the behavior of the system right before and after the pulse. The system of difference equations is called the stroboscopic map, where equilibria of the stroboscopic map correspond to periodic solutions in the system. The most difficult part in the stability analysis is as the number of systems of impulsive equations increase, it is difficult to obtain manageable formula for the stroboscopic map.

Therefore, in this paper, a novel dynamic system for HPV disease transmission where impulsive vaccination is implemented at regular intervals for both the susceptible female and male population is proposed. The main aim is to investigate the impulsive vaccine control strategy for deciding whether or not the disease dies out and to explore how the control strategy affects HPV prevention and control. The impact of the pulse vaccination on both the infected female and male population is discussed. Moreover, we found the critical value of the pulse vaccination for susceptible females. Also, the time series of vaccinated females and males are compared graphically to see the effect of the implementation of the pulse vaccination. It is assumed that both vaccinated and infected can get infected again. The remainder of this paper is structured in the following way. The model formulation and its analysis are presented in Sect. 2. Within this, we present the basic qualitative analysis and some basic concepts on the stroboscopic map. Section 3 presents the nature of the disease-free equilibrium and its global stability. Section 4 studies the uniform persistence of the disease. In Sect. 5, numerical simulation and discussion are presented. The conclusion and limitations of the study are given in the last section.

\section{Model formulation and analysis}

We assume the heterosexually active population is divided into compartments according to gender (male or female), state of disease (susceptible or infected), and status of immunization (vaccinated or unvaccinated). The total female population is divided into mutually exclusive compartments, including susceptible unvaccinated females $S_{\mathrm{uf}}$, infected females $I_{\mathrm{f}}$, and susceptible vaccinated females $S_{\mathrm{vf}}$. The total female population being given as

$N_{\mathrm{f}}(t)=S_{\mathrm{uf}}(t)+I_{\mathrm{f}}(t)+S_{\mathrm{vf}}(t)$.

The total male population is divided into mutually exclusive compartments, including susceptible unvaccinated males $S_{\text {um }}$, infected males $I_{\mathrm{m}}$, and susceptible 
vaccinated males $S_{\mathrm{vm}}$. The total male population being given as

$N_{\mathrm{m}}(t)=S_{\mathrm{um}}(t)+I_{\mathrm{m}}(t)+S_{\mathrm{vm}}(t)$.

The force of infection for the female and male population is assumed to be pseudo-mass-action. We assume that female and male individuals join the sexually active population at a constant rate of $\Pi_{\mathrm{f}}$ and $\Pi_{\mathrm{m}}$, respectively. In this study, we have assumed two types of vaccination strategies named by prior and after sexual initiation. Particularly, a fraction $p_{1}$ of female children and a fraction $p_{2}$ of male children are vaccinated before joining the sexually active class and are thus recruited into their vaccinated compartment. Moreover, the sexually active susceptible unvaccinated female and the male population is assumed to be given an impulsive vaccination periodically. The vaccine-induced immunity for both sex groups is supposed to wane at a rate of $\theta$.

Susceptible unvaccinated females may develop an infection at per-capita rate $\beta_{\mathrm{m}} I_{\mathrm{m}}(t)$ due to the sexual act rate of $\left(1-\psi_{2}\right) \beta_{\mathrm{f}} I_{\mathrm{f}}(t)$ (where $\psi_{2}$ is the level of protection due to the vaccine where $0 \leq \psi_{2} \leq 1$, and $\psi_{2}=0$ means no protection). $\beta_{\mathrm{f}}$ and $\beta_{\mathrm{m}}$ represent the average number of adequate contacts of an infectious female and male individuals, respectively per unit time. Females and males leave the population by natural death or ceasing sexual activity at per-capita levels $\mu_{\mathrm{f}}$ and $\mu_{\mathrm{m}}$, respectively. Infectious hosts experience an extra disease-related death at a constant rate of $d_{\mathrm{f}}$ for females and $d_{\mathrm{m}}$ for males. The recovered female and male individuals will lose immunity at a rate of $\gamma_{\mathrm{f}}$ and $\gamma_{\mathrm{m}}$, respectively. $\theta_{1}\left(0<\theta_{1}<1\right)$ and $\theta_{2}\left(0<\theta_{2}<1\right)$ represent the proportion of female and male who are vaccinated successfully, which is called pulse vaccination duration, and $T$ is the interpulse time, that is, the time between two consecutive pulse vaccinations. Following the implementation of each pulse vaccination, the system evolves by the standard SIVS model until next pulse is applied at time $t_{n+1}=t_{n}+T$.

The system of ordinary differential equations capturing such dynamics is given as:

$$
\left\{\begin{aligned}
\frac{\mathrm{d} S_{\mathrm{uf}}(t)}{\mathrm{d} t} & =\left(1-p_{1}\right) \Pi_{\mathrm{f}}+\gamma_{\mathrm{f}} I_{\mathrm{f}}(t)+\theta S_{\mathrm{vf}}(t)-\left(\mu_{\mathrm{f}}+\beta_{\mathrm{m}} I_{\mathrm{m}}(t)\right) S_{\mathrm{uf}}(t), \\
\frac{\mathrm{d} I_{\mathrm{f}}(t)}{\mathrm{d} t} & =\left(S_{\mathrm{uf}}(t)+\left(1-\Psi_{1}\right) S_{\mathrm{vf}}(t)\right) \beta_{\mathrm{m}} I_{\mathrm{m}}(t)-\left(\mu_{\mathrm{f}}+\gamma_{\mathrm{f}}+d_{\mathrm{f}}\right) I_{\mathrm{f}}(t), \\
\frac{\mathrm{d} S_{\mathrm{vf}}(t)}{\mathrm{d} t} & =p_{1} \Pi_{\mathrm{f}}-\left(\theta+\mu_{\mathrm{f}}+\left(1-\Psi_{1}\right) \beta_{\mathrm{m}} I_{\mathrm{m}}(t)\right) S_{\mathrm{vf}}(t), \\
\frac{\mathrm{d} S_{\mathrm{um}}(t)}{\mathrm{d} t} & =\left(1-p_{2}\right) \Pi_{\mathrm{m}}+\gamma_{\mathrm{m}} I_{\mathrm{m}}(t)+\theta S_{\mathrm{vm}}(t)-\left(\mu_{\mathrm{m}}+\beta_{\mathrm{f}} I_{\mathrm{f}}(t)\right) S_{\mathrm{um}}(t), \\
\frac{\mathrm{d} I_{\mathrm{m}}(t)}{\mathrm{d} t} & =\left(S_{\mathrm{um}}(t)+\left(1-\Psi_{2}\right) S_{\mathrm{vm}}(t)\right) \beta_{\mathrm{f}} I_{\mathrm{f}}(t)-\left(\mu_{\mathrm{m}}+\gamma_{\mathrm{m}}+d_{\mathrm{m}}\right) I_{\mathrm{m}}(t), \\
\frac{\mathrm{d} S_{\mathrm{vm}}(t)}{\mathrm{d} t} & =p_{2} \Pi_{\mathrm{m}}-\left(\theta+\mu_{\mathrm{m}}+\left(1-\Psi_{2}\right) \beta_{\mathrm{f}} I_{\mathrm{f}}(t)\right) S_{\mathrm{vm}}(t), \\
S_{\mathrm{uf}}\left(t^{+}\right) & =\left(1-\theta_{1}\right) S_{\mathrm{uf}}(t), \\
I_{\mathrm{f}}\left(t^{+}\right) & =I_{\mathrm{f}}(t), \\
S_{\mathrm{vf}}\left(t^{+}\right) & =S_{\mathrm{vf}}(t)+\theta_{1} S_{\mathrm{uf}}(t), \\
S_{\mathrm{um}}\left(t^{+}\right) & =\left(1-\theta_{2}\right) S_{\mathrm{um}}(t), \\
I_{\mathrm{m}}\left(t^{+}\right) & =I_{\mathrm{m}}(t), \\
S_{\mathrm{vm}}\left(t^{+}\right) & =S_{\mathrm{vm}}(t)+\theta_{2} S_{\mathrm{um}}(t),
\end{aligned}\right\} \quad t=n T, \quad n
$$

with the infected unvaccinated and infected vaccinated males. Susceptible vaccinated females get infected at a reduced rate of $\left(1-\psi_{1}\right) \beta_{\mathrm{m}} I_{\mathrm{m}}(t)$ (where $\psi_{1}$ is the level of protection due to the vaccine where $0 \leq \psi_{1} \leq 1$, and $\psi_{1}=0$ means no protection). Susceptible unvaccinated males get infected if they have sexual acts with infected females at a per-capita rate of $\beta_{\mathrm{f}} I_{\mathrm{f}}(t)$. Susceptible vaccinated males get infected at a reduced
$S_{\mathrm{uf}}(t)=S_{\mathrm{uf}}\left(t^{-}\right), I_{\mathrm{f}}(t)=I_{\mathrm{f}}\left(t^{-}\right), S_{\mathrm{vf}}(t)=S_{\mathrm{vf}}\left(t^{-}\right)$ are defined as the numbers of susceptible unvaccinated females, infected females, and vaccinated susceptible females, respectively, just before the pulse. Moreover, $S_{\mathrm{um}}(t)=S_{\mathrm{um}}\left(t^{-}\right), I_{\mathrm{m}}(t)=I_{\mathrm{m}}\left(t^{-}\right), S_{\mathrm{vm}}(t)=$ $S_{\mathrm{vm}}\left(t^{-}\right)$are defined as the numbers of susceptible unvaccinated males, infected males, vaccinated susceptible males before the pulse. On the other hand, $S_{\mathrm{uf}}\left(t^{+}\right)$, 
$I_{\mathrm{f}}\left(t^{+}\right)$and $S_{\mathrm{vf}}\left(t^{+}\right)$are defined as the new numbers of susceptible unvaccinated, infected, and susceptible vaccinated females immediately after the pulse. And $S_{\mathrm{um}}\left(t^{+}\right), I_{\mathrm{m}}\left(t^{+}\right)$and $S_{\mathrm{vm}}\left(t^{+}\right)$are defined as the new numbers of susceptible unvaccinated, infected, susceptible vaccinated males, respectively. Therefore, equations from seventh to twelfth of system Eq. (1) means we have used a new vaccination for the susceptible unvaccinated people $S_{\mathrm{uf}}(t)$ and $S_{\mathrm{um}}(t)$.

The total female population size $N_{\mathrm{f}}(t)=S_{\mathrm{uf}}(t)+$ $I_{\mathrm{f}}(t)+S_{\mathrm{vf}}(t)$ can form the differential equation

$\frac{\mathrm{d} N_{\mathrm{f}}(t)}{\mathrm{d} t}=\Pi_{\mathrm{f}}-\mu_{\mathrm{f}} N_{\mathrm{f}}(t)-d_{\mathrm{f}} I_{\mathrm{f}}(t)$,

by adding the first three equations of system Eq. (1). This implies the total female population may vary in time. From Eq. (2), we have $\Pi_{\mathrm{f}}-\left(\mu_{\mathrm{f}}+d_{\mathrm{f}}\right) N_{\mathrm{f}}(t) \leq$ $\frac{\mathrm{d} N_{\mathrm{f}}(t)}{\mathrm{d} t} \leq \Pi_{\mathrm{f}}-\mu_{\mathrm{f}} N_{\mathrm{f}}(t)$. It follows that

$$
\left\{\begin{aligned}
\frac{\mathrm{d} S_{\mathrm{uf}}(t)}{\mathrm{d} t} & =\left(1-p_{1}\right) \Pi_{\mathrm{f}}+\gamma_{\mathrm{f}} I_{\mathrm{f}}(t)+\theta S_{\mathrm{vf}}(t)-\left(\mu_{\mathrm{f}}+\beta_{\mathrm{m}} I_{\mathrm{m}}(t)\right) S_{\mathrm{uf}}(t), \\
\frac{\mathrm{d} I_{\mathrm{f}}(t)}{\mathrm{d} t} & =\left(S_{\mathrm{uf}}(t)+\left(1-\Psi_{1}\right) S_{\mathrm{vf}}(t)\right) \beta_{\mathrm{m}} I_{\mathrm{m}}(t)-\left(\mu_{\mathrm{f}}+\gamma_{\mathrm{f}}+d_{\mathrm{f}}\right) I_{\mathrm{f}}(t), \\
\frac{\mathrm{d} S_{\mathrm{vf}}(t)}{\mathrm{d} t} & =p_{1} \Pi_{\mathrm{f}}-\left(\theta+\mu_{\mathrm{f}}+\left(1-\Psi_{1}\right) \beta_{\mathrm{m}} I_{\mathrm{m}}(t)\right) S_{\mathrm{vf}}(t), \\
\frac{\mathrm{d} N_{\mathrm{f}}}{\mathrm{d} t} & =\Pi_{\mathrm{f}}-\mu_{\mathrm{f}} N_{\mathrm{f}}(t)-d_{\mathrm{f}} I_{\mathrm{f}}(t), \\
\frac{\mathrm{d} S_{\mathrm{um}}(t)}{\mathrm{d} t} & =\left(1-p_{2}\right) \Pi_{\mathrm{m}}+\gamma_{\mathrm{m}} I_{\mathrm{m}}(t)+\theta S_{\mathrm{vm}}(t)-\left(\mu_{\mathrm{m}}+\beta_{\mathrm{f}} I_{\mathrm{f}}(t)\right) S_{\mathrm{um}}(t), \\
\frac{\mathrm{d} I_{\mathrm{m}}(t)}{\mathrm{d} t} & =\left(S_{\mathrm{um}}(t)+\left(1-\Psi_{2}\right) S_{\mathrm{vm}}(t)\right) \beta_{\mathrm{f}} I_{\mathrm{f}}(t)-\left(\mu_{\mathrm{m}}+\gamma_{\mathrm{m}}+d_{\mathrm{m}}\right) I_{\mathrm{m}}(t), \\
\frac{\mathrm{d} S_{\mathrm{vm}}(t)}{\mathrm{d} t} & =p_{2} \Pi_{\mathrm{m}}-\left(\theta+\mu_{\mathrm{m}}+\left(1-\Psi_{2}\right) \beta_{\mathrm{f}} I_{\mathrm{f}}(t)\right) S_{\mathrm{vm}}(t), \\
\frac{\mathrm{d} N_{\mathrm{m}}}{\mathrm{d} t} & =\Pi_{\mathrm{m}}-\mu_{\mathrm{m}} N_{\mathrm{m}}(t)-d_{\mathrm{m}} I_{\mathrm{m}}(t), \\
S_{\mathrm{uf}}\left(t^{+}\right) & =\left(1-\theta_{1}\right) S_{\mathrm{uf}}(t), \\
I_{\mathrm{f}}\left(t^{+}\right) & =I_{\mathrm{f}}(t), \\
S_{\mathrm{vf}}\left(t^{+}\right) & =S_{\mathrm{vf}}(t)+\theta_{1} S_{\mathrm{uf}}(t), \\
N_{\mathrm{f}}\left(t^{+}\right) & =N_{\mathrm{f}}(t), \\
S_{\mathrm{um}}\left(t^{+}\right) & =\left(1-\theta_{2}\right) S_{\mathrm{um}}(t), \\
I_{\mathrm{m}}\left(t^{+}\right) & =I_{\mathrm{m}}(t), \\
S_{\mathrm{vm}}\left(t^{+}\right) & =S_{\mathrm{vm}}(t)+\theta_{2} S_{\mathrm{um}}(t), \\
N_{\mathrm{f}}\left(t^{+}\right) & =N_{\mathrm{f}}(t)
\end{aligned}\right\} \quad t=n T, n=1,2,3, \ldots .
$$


The initial condition of system Eq. (4) is given as

$$
\begin{aligned}
& \left(S_{\mathrm{uf} 0}, I_{\mathrm{f} 0}, S_{\mathrm{vf} 0}, N_{\mathrm{f} 0}, S_{\mathrm{um} 0}, I_{m o}, S_{\mathrm{vm} 0}, N_{\mathrm{m} 0}\right) \in \mathrm{R}_{+}^{6} \\
& \quad=\left\{\left(x_{1}, x_{2}, x_{3}, x_{4}, x_{5}, x_{6}, x_{7}, x_{8}\right): x_{i} \geq 0, i\right. \\
& \quad=1,2,3,4,5,6,7,8\} .
\end{aligned}
$$

For biological seasons, we analyze the complex behavior of the model Eq. (4) in the following closed set are continuous at $t \in[0,+\infty)$. Since there is a limit to $N_{\mathrm{f}}(t)$ and $N_{\mathrm{m}}(t)$, so $N_{\mathrm{f}}(t)$ and $N_{\mathrm{m}}(t)$ are uniformly ultimately bounded. So, by the concept of $N_{\mathrm{f}}(t)$ and $N_{\mathrm{m}}(t)$, we get that there is a positive integer $n_{01}$ such that $S_{\mathrm{uf}}(t) \leq \frac{\Pi_{\mathrm{f}}}{\mu_{\mathrm{f}}}, I_{\mathrm{f}}(t) \leq \frac{\Pi_{\mathrm{f}}}{\mu_{\mathrm{f}}}, S_{\mathrm{vf}}(t) \leq \frac{\Pi_{\mathrm{f}}}{\mu_{\mathrm{f}}}$ and $S_{\mathrm{um}}(t) \leq \frac{\Pi_{\mathrm{m}}}{\mu_{\mathrm{m}}}, I_{\mathrm{f}}(t) \leq \frac{\Pi_{\mathrm{m}}}{\mu_{\mathrm{m}}}, S_{\mathrm{vm}}(t) \leq \frac{\Pi_{\mathrm{m}}}{\mu_{\mathrm{m}}}$ for all $t \geq n_{01} T$.

$\Omega=\left\{\left(S_{\mathrm{uf}}(t), I_{\mathrm{f}}(t), S_{\mathrm{vf}}(t), N_{\mathrm{f}}(t), S_{\mathrm{um}}(t), I_{\mathrm{m}}(t), S_{\mathrm{vm}}(t), N_{\mathrm{m}}(t)\right) \in \mathrm{R}_{+}^{8}: 0 \leq N_{\mathrm{f}}(t) \leq \frac{\Pi_{\mathrm{f}}}{\mu_{\mathrm{f}}}, 0 \leq N_{\mathrm{m}}(t) \leq \frac{\Pi_{\mathrm{m}}}{\mu_{\mathrm{m}}}\right\}$.

$\mathrm{R}_{+}^{8}$ depicts the nonnegative cone of $\mathrm{R}^{8}$ with its lower dimensional faces.

Lemma 2.1 Every component of any solution of system Eq. (4) is nonnegative, and the closed set $\Omega$ is positively invariant and attracting with respect to Eq. (4).

Proof We know from above that the total human population is not constant. Consequently,

$$
\begin{aligned}
\frac{\mathrm{d} N_{\mathrm{f}}(t)}{\mathrm{d} t} & \leq \Pi_{\mathrm{f}}-\mu_{\mathrm{f}} N_{\mathrm{f}}(t) \text { and } \frac{\mathrm{d} N_{\mathrm{m}}(t)}{\mathrm{d} t} \\
& \leq \Pi_{\mathrm{m}}-\mu_{\mathrm{m}} N_{\mathrm{m}}(t) .
\end{aligned}
$$

It follows that

$$
\begin{aligned}
N_{\mathrm{f}}(t) & \leq\left(N_{\mathrm{f} 0}-\frac{\Pi_{\mathrm{f}}}{\mu_{\mathrm{f}}}\right) e^{-\mu_{\mathrm{f}} t}+\frac{\Pi_{\mathrm{f}}}{\mu_{\mathrm{f}}} \text { and } N_{\mathrm{m}}(t) \\
& \leq\left(N_{\mathrm{m} 0}-\frac{\Pi_{\mathrm{m}}}{\mu_{\mathrm{m}}}\right) e^{-\mu_{\mathrm{m}} t}+\frac{\Pi_{\mathrm{m}}}{\mu_{\mathrm{m}}}
\end{aligned}
$$

From Eq. (6), we observe that $N_{\mathrm{f}}(t) \rightarrow \frac{\Pi_{\mathrm{f}}}{\mu_{\mathrm{f}}}$ and $N_{\mathrm{m}}(t) \rightarrow \frac{\Pi_{\mathrm{m}}}{\mu_{\mathrm{m}}}$ as $t \rightarrow \infty$. We have two implications. If $N_{\mathrm{f} 0} \geq \frac{\Pi_{\mathrm{f}}}{\mu_{\mathrm{f}}}$ and $N_{\mathrm{m} 0} \geq \frac{\Pi_{\mathrm{m}}}{\mu_{\mathrm{m}}}$ then $\lim _{t \rightarrow \infty} N_{\mathrm{f}}(t)=\frac{\Pi_{\mathrm{f}}}{\mu_{\mathrm{f}}}$ and $\lim _{t \rightarrow \infty} N_{\mathrm{m}}(t)=\frac{\Pi_{\mathrm{m}}}{\mu_{\mathrm{m}}}$. In this case, $\frac{\Pi_{\mathrm{f}}}{\mu_{\mathrm{f}}}$ is the upper bound of $N_{\mathrm{f}}(t)$ and $\frac{\Pi_{\mathrm{m}}}{\mu_{\mathrm{m}}}$ is the upper bound of $N_{\mathrm{m}}(t)$. On the other hand, if $N_{\mathrm{f} 0}>\frac{\Pi_{\mathrm{f}}}{\mu_{\mathrm{f}}}$ and $N_{\mathrm{m} 0}>$ $\frac{\Pi_{\mathrm{m}}}{\mu_{\mathrm{m}}}$, then the solutions $N_{\mathrm{f}}(t)$ and $N_{\mathrm{m}}(t)$ will decrease to $\frac{\Pi_{\mathrm{f}}}{\mu_{\mathrm{f}}}$ and $\frac{\Pi_{\mathrm{m}}}{\mu_{\mathrm{m}}}$, respectively as $t \rightarrow \infty$. Denoting $M:=\max \left\{\frac{\Pi_{\mathrm{f}}}{\mu_{\mathrm{f}}}, \frac{\Pi_{\mathrm{m}}}{\mu_{\mathrm{m}}}\right\}$ we conclude that $\Omega$ is positively invariant. Then either the solution enters $\Omega$ infinite time or $\limsup _{t \rightarrow \infty} N_{\mathrm{f}}(t) \leq \frac{\Pi_{\mathrm{f}}}{\mu_{\mathrm{f}}} \leq M$ and $\lim \sup _{t \rightarrow \infty} N_{\mathrm{m}}(t) \leq \frac{\Pi_{\mathrm{m}}}{\mu_{\mathrm{m}}} \leq M$, respectively. Hence, the region $\Omega$ attracts all solutions.

Because $N_{\mathrm{f}}\left(t^{+}\right)=N_{\mathrm{f}}\left(t^{-}\right)=N_{\mathrm{f}}(t)$ and $N_{\mathrm{m}}\left(t^{+}\right)=$ $N_{\mathrm{m}}\left(t^{-}\right)=N_{\mathrm{m}}(t)$ for all $t \geq 0$, then $N_{\mathrm{f}}(t)$ and $N_{\mathrm{m}}(t)$
Next, we state the following Lemma for the solution of general impulsive differential equations, which is useful for our main results.

\section{Lemma 2.2}

$\left\{\begin{array}{l}\frac{\mathrm{d} u(t)}{\mathrm{d} t}=a-b u(t), \quad t \neq n T, \\ u\left(t^{+}\right)=(1-\theta) u(t), \quad t=n T,\end{array}\right.$

where $a>0, b>0$, and $0<\theta<1$. There exists a globally asymptotically stable periodic solution for system Eq. (7) which is:

$u_{p}(t)=\frac{a}{b}+\left(u^{*}-\frac{a}{b}\right) e^{-b(t-n T)}, \quad n T<t \leq(n+1) T$,

where

$$
u^{*}=\frac{a(1-\theta)\left(1-e^{-b T}\right)}{b\left(1-(1-\theta) e^{-b T}\right)} .
$$

Proof Integrating and solving the first equation of Eq. (7) between pulses results in

$$
\begin{aligned}
u_{p}(t) & =\frac{a}{b}+\left(u(n T)-\frac{a}{b}\right) e^{-b(t-n T)}, \\
n T & <t \leq(n+1) T,
\end{aligned}
$$

where $u(n T)$ is the initial value at time $n T$. Using the second equation of system Eq. (7), we deduce the stroboscopic map such that

$$
\begin{aligned}
& u((n+1) T)=(1-\theta)\left(\frac{a}{b}+\left(u(n T)-\frac{a}{b}\right) e^{-b T}\right) \\
& \quad:=f(u(n T))
\end{aligned}
$$

where $f(u)=(1-\theta)\left(\frac{a}{b}+\left(u-\frac{a}{b}\right) e^{-b T}\right)$. Equation (10) has a unique positive equilibrium. In fact, assume that $u^{*}$ be the positive equilibrium of Eq. (10); then $u^{*}=u((n+1) T)=u(n T)$, and it satisfies

$u^{*}=(1-\theta)\left(\frac{a}{b}+\left(u^{*}-\frac{a}{b}\right) e^{-b T}\right)$. 
satisfy the following systems of impulsive differential equations:

$$
\left\{\begin{aligned}
\frac{\mathrm{d} S_{\mathrm{uf}}(t)}{\mathrm{d} t} & =\left(1-p_{1}\right) \Pi_{\mathrm{f}}+\theta S_{\mathrm{vf}}(t)-\mu_{\mathrm{f}} S_{\mathrm{uf}}(t), \\
\frac{\mathrm{d} S_{\mathrm{vf}}(t)}{\mathrm{d} t} & =p_{1} \Pi_{\mathrm{f}}-\left(\theta+\mu_{\mathrm{f}}\right) S_{\mathrm{vf}}(t), \\
\frac{\mathrm{d} N_{\mathrm{f}}(t)}{\mathrm{d} t} & =\Pi_{\mathrm{f}}-\mu_{\mathrm{f}} N_{\mathrm{f}}(t), \\
\frac{\mathrm{d} S_{\mathrm{um}}(t)}{\mathrm{d} t} & =\left(1-p_{2}\right) \Pi_{\mathrm{m}}+\theta S_{\mathrm{vm}}(t)-\mu_{\mathrm{m}} S_{\mathrm{um}}(t), \\
\frac{\mathrm{d} S_{\mathrm{vm}}(t)}{\mathrm{d} t} & =p_{2} \Pi_{\mathrm{m}}-\left(\theta+\mu_{\mathrm{m}}\right) S_{\mathrm{vm}}(t), \\
\frac{\mathrm{d} N_{\mathrm{m}}(t)}{\mathrm{d} t} & =\Pi_{\mathrm{m}}-\mu_{\mathrm{m}} N_{\mathrm{m}}(t), \\
S_{\mathrm{uf}}\left(t^{+}\right) & =\left(1-\theta_{1}\right) S_{\mathrm{uf}}(t), \\
S_{\mathrm{vf}}\left(t^{+}\right) & =S_{\mathrm{vf}}(t)+\theta_{1} S_{\mathrm{uf}}(t), \\
N_{\mathrm{f}}\left(t^{+}\right) & =N_{\mathrm{f}}(t), \\
S_{\mathrm{um}}\left(t^{+}\right) & =\left(1-\theta_{2}\right) S_{\mathrm{um}}(t), \\
S_{\mathrm{vm}}\left(t^{+}\right) & =S_{\mathrm{vm}}(t)+\theta_{2} S_{\mathrm{um}}(t), \\
N_{\mathrm{f}}\left(t^{+}\right) & =N_{\mathrm{f}}(t)
\end{aligned}\right\} \quad t=n T, n \in \mathbb{N}
$$

Solving this we obtain the unique positive equilibrium $u^{*}=\frac{a}{b} \frac{(1-\theta)\left(1-e^{-b T}\right)}{\left(1-(1-\theta) e^{-b T}\right)}$. From system Eq. (10), we obtain that $f^{\prime}(u)=(1-\theta) e^{-b T}$. It is apparent that $0<f^{\prime}(u)<1$; then $0<f\left(u^{*}\right)<1$; thus, $u^{*}$ is a stable positive equilibrium of Eq. (10). Substituting the expression of $u^{*}$ into Eq. (9), we obtain

$u_{p}(t)=\frac{a}{b}+\left(u^{*}-\frac{a}{b}\right) e^{-b(t-n T)}, \quad n T<t \leq(n+1) T$.

We obtained that $u^{*}$ is the unique positive equilibrium of the difference equations, then $u_{p}(t)$ is the unique global asymptotically stable periodic solution of the system Eq. (7). This completes the proof.

\section{The global attractivity of disease-free periodic solution}

If there is no infection, then $I_{\mathrm{f}}(t)=I_{\mathrm{m}}(t)=0$ for all $t \geq 0$. In such case, the dynamics of the susceptible unvaccinated, vaccinated and total population must
From the third and ninth equations of Eq. (11), we easily obtain $\lim _{t \rightarrow \infty} N_{\mathrm{f}}(t)=\frac{\Pi_{\mathrm{f}}}{\mu_{\mathrm{f}}}$. Moreover, if $I_{\mathrm{m}}(t)=0$, it follows from the sixth and the last equations that $\lim _{t \rightarrow \infty} N_{\mathrm{m}}(t)=\frac{\Pi_{\mathrm{m}}}{\mu_{\mathrm{m}}}$. In the following, we show that the susceptible unvaccinated and the vaccinated population oscillate with period $T$, in accordance with the periodic pulse vaccination. In view of the value of $\lim _{t \rightarrow \infty} N_{\mathrm{f}}(t)=\frac{\Pi_{\mathrm{f}}}{\mu_{\mathrm{f}}}$ and $\lim _{t \rightarrow \infty} N_{\mathrm{m}}(t)=\frac{\Pi_{\mathrm{m}}}{\mu_{\mathrm{m}}}$, we have the following limit results from system Eq. (11)

$S_{\mathrm{vf}}(t)=\frac{\Pi_{\mathrm{f}}}{\mu_{\mathrm{f}}}-S_{\mathrm{uf}}(t)$ and $S_{\mathrm{vm}}(t)=\frac{\Pi_{\mathrm{m}}}{\mu_{\mathrm{m}}}-S_{\mathrm{um}}(t)$.

Under this condition, the growth of susceptible unvaccinated, susceptible vaccinated and the total population satisfy the following impulsive system: 


$$
\begin{aligned}
& \int \frac{\mathrm{d} S_{\mathrm{uf}}(t)}{\mathrm{d} t}=\left(1-p_{1}+\frac{\theta}{\mu_{\mathrm{f}}}\right) \Pi_{\mathrm{f}}-\left(\mu_{\mathrm{f}}+\theta\right) S_{\mathrm{uf}}(t), \\
& \frac{\mathrm{d} S_{\mathrm{vf}}(t)}{\mathrm{d} t}=p_{1} \Pi_{\mathrm{f}}-\left(\theta+\mu_{\mathrm{f}}\right) S_{\mathrm{vf}}(t), \\
& \frac{\mathrm{d} S_{\mathrm{um}}(t)}{\mathrm{d} t}=\left(1-p_{2}+\frac{\theta}{\mu_{\mathrm{m}}}\right) \Pi_{\mathrm{m}}-\left(\mu_{\mathrm{m}}+\theta\right) S_{\mathrm{um}}(t), \\
& \frac{\mathrm{d} S_{\mathrm{vm}}(t)}{\mathrm{d} t}=p_{2} \Pi_{\mathrm{m}}-\left(\theta+\mu_{\mathrm{m}}\right) S_{\mathrm{vm}}(t), \\
& S_{\mathrm{uf}}\left(t^{+}\right)=\left(1-\theta_{1}\right) S_{\mathrm{uf}}(t) \text {, } \\
& S_{\mathrm{vf}}\left(t^{+}\right)=S_{\mathrm{vf}}(t)+\theta_{1} S_{\mathrm{uf}}(t) \text {, } \\
& S_{\mathrm{um}}\left(t^{+}\right)=\left(1-\theta_{2}\right) S_{\mathrm{um}}(t) \text {, } \\
& t=n T, n \in \mathbb{N} \text {. } \\
& t \neq n T, n \in \mathbb{N} \\
& S_{\mathrm{vm}}\left(t^{+}\right)=S_{\mathrm{vm}}(t)+\theta_{2} S_{\mathrm{um}}(t), \text { ) }
\end{aligned}
$$

Following Lemma 2.2, it is easy to obtain the following periodic solution of system Eq. (13), which is globally asymptotically stable.

$$
\begin{aligned}
& \bar{S}_{\mathrm{ufe}}(t)=\frac{\left(\left(1-p_{1}\right) \mu_{\mathrm{f}}+\theta\right) \Pi_{\mathrm{f}}}{\mu_{\mathrm{f}}\left(\theta+\mu_{\mathrm{f}}\right)} \\
& +\left(S_{\mathrm{uf} 0}-\frac{\left(\left(1-p_{1}\right) \mu_{\mathrm{f}}+\theta\right) \Pi_{\mathrm{f}}}{\mu_{\mathrm{f}}\left(\theta+\mu_{\mathrm{f}}\right)}\right) \\
& e^{-\left(\mu_{\mathrm{f}}+\theta\right)(t-n T)}, \\
& \bar{S}_{\mathrm{vf}}(t)=\frac{p_{1} \Pi_{\mathrm{f}}}{\mu_{\mathrm{f}}+\theta} \\
& +\left(S_{\mathrm{vf} 0}-\frac{p_{1} \Pi_{\mathrm{f}}}{\mu_{\mathrm{f}}+\theta}\right) e^{-\left(\mu_{\mathrm{f}}+\theta\right)(t-n T)}, \\
& \bar{S}_{\mathrm{ume}}(t)=\frac{\left(\left(1-p_{2}\right) \mu_{\mathrm{m}}+\theta\right) \Pi_{\mathrm{m}}}{\mu_{\mathrm{m}}\left(\theta+\mu_{\mathrm{m}}\right)} \\
& +\left(S_{\mathrm{um} 0}-\frac{\left(\left(1-p_{2}\right) \mu_{\mathrm{m}}+\theta\right) \Pi_{\mathrm{m}}}{\mu_{\mathrm{m}}\left(\theta+\mu_{\mathrm{m}}\right)}\right) \\
& e^{-\left(\mu_{\mathrm{m}}+\theta\right)(t-n T)}, n T<t \leq(n+1) T, \\
& \bar{S}_{\mathrm{vm}}(t)=\frac{p_{2} \Pi_{\mathrm{m}}}{\mu_{\mathrm{m}}+\theta}+\left(S_{\mathrm{vm} 0}-\frac{p_{2} \Pi_{\mathrm{m}}}{\mu_{\mathrm{m}}+\theta}\right) \\
& e^{-\left(\mu_{\mathrm{m}}+\theta\right)(t-n T)},
\end{aligned}
$$

where

$$
\begin{aligned}
S_{\mathrm{uf} 0} & =\frac{\Pi_{\mathrm{f}}\left(\left(1-p_{1}\right) \mu_{\mathrm{f}}+\theta\right)\left(1-\theta_{1}\right)\left(1-e^{-\left(\mu_{\mathrm{f}}+\theta\right) T}\right)}{\mu_{\mathrm{f}}\left(\mu_{\mathrm{f}}+\theta\right)\left(1-\left(1-\theta_{1}\right) e^{-\left(\mu_{\mathrm{f}}+\theta\right) T}\right)}, \\
S_{\mathrm{vf} 0} & =\frac{p_{1} \Pi_{\mathrm{f}}}{\mu_{\mathrm{f}}+\theta}+\theta_{1} \frac{\Pi_{\mathrm{f}}\left(\left(1-p_{1}\right) \mu_{\mathrm{f}}+\theta\right)}{\mu_{\mathrm{f}}\left(\theta+\mu_{\mathrm{f}}\right)\left(1-\left(1-\theta_{1}\right) e^{-\left(\mu_{\mathrm{f}}+\theta\right) T}\right)}, \\
S_{\mathrm{um} 0} & =\frac{\Pi_{\mathrm{m}}\left(\left(1-p_{2}\right) \mu_{\mathrm{m}}+\theta\right)\left(1-\theta_{2}\right)\left(1-e^{-\left(\mu_{\mathrm{m}}+\theta\right) T}\right)}{\mu_{\mathrm{m}}\left(\mu_{\mathrm{m}}+\theta\right)\left(1-\left(1-\theta_{2}\right) e^{-\left(\mu_{\mathrm{m}}+\theta\right) T}\right)}, \\
S_{\mathrm{vm} 0} & =\frac{p_{2} \Pi_{\mathrm{m}}}{\mu_{\mathrm{m}}+\theta}+\theta_{2} \frac{\Pi_{\mathrm{m}}\left(\left(1-p_{2}\right) \mu_{\mathrm{m}}+\theta\right)}{\mu_{\mathrm{m}}\left(\theta+\mu_{\mathrm{m}}\right)\left(1-\left(1-\theta_{2}\right) e^{-\left(\mu_{\mathrm{m}}+\theta\right) T}\right)} .
\end{aligned}
$$

In the following theorem, we determine the global attractivity condition of the disease-free periodic solution:
$\left(\bar{S}_{\text {ufe }}(t), 0, \frac{\Pi_{\mathrm{f}}}{\mu_{\mathrm{f}}}-\bar{S}_{\text {ufe }}(t), \bar{S}_{\text {ume }}(t), 0, \frac{\Pi_{\mathrm{m}}}{\mu_{\mathrm{m}}}-\bar{S}_{\text {ume }}(t)\right)$. We define the reproduction number $R_{0}$ as:

$$
\begin{aligned}
& R_{0}=\frac{1}{M^{*} T} \int_{0}^{T}\left(\beta_{\mathrm{m}}\left(\bar{S}_{\mathrm{ufe}}(t)+\left(1-\Psi_{1}\right) \bar{S}_{\mathrm{vfe}}(t)\right)\right. \\
& \left.\quad+\beta_{\mathrm{f}}\left(\bar{S}_{\text {ume }}(t)+\left(1-\Psi_{2}\right) \bar{S}_{\text {vme }}(t)\right)\right) \mathrm{d} t
\end{aligned}
$$

where $M^{*}=\min \left\{\mu_{\mathrm{f}}+d_{\mathrm{f}}+\gamma_{\mathrm{f}}, \mu_{\mathrm{m}}+d_{\mathrm{m}}+\gamma_{\mathrm{m}}\right\}$.

Theorem 3.1 The disease-free periodic solution $\left(\bar{S}_{\text {ufe }}(t), 0, \frac{\Pi_{\mathrm{f}}}{\mu_{\mathrm{f}}}-\bar{S}_{\text {ufe }}(t), \bar{S}_{\text {ume }}(t), 0, \frac{\Pi_{\mathrm{m}}}{\mu_{\mathrm{m}}}-\bar{S}_{\text {ume }}(t)\right)$ of system Eq. (4) is globally attractive provided that $R_{0}<1$.

Proof Since $R_{0}<1$, we can choose $\epsilon>0$ sufficiently small such that

$$
\begin{aligned}
& \int_{0}^{T}\left(\beta_{\mathrm{m}}\left(\bar{S}_{\mathrm{ufe}}(t)+\left(1-\Psi_{1}\right) \bar{S}_{\mathrm{vfe}}(t)\right)\right. \\
& \quad+\beta_{\mathrm{f}}\left(\bar{S}_{\mathrm{ume}}(t)+\left(1-\Psi_{2}\right) \bar{S}_{\mathrm{vme}}(t)\right)+\left(\left(2-\Psi_{1}\right) \beta_{\mathrm{m}}\right. \\
&\left.\left.\quad+\left(2-\Psi_{2}\right) \beta_{\mathrm{f}}\right) \epsilon-M^{*}\right) \mathrm{d} t
\end{aligned}
$$

From the first, third, fifth, and sixth equations of system Eq. (4), we have $\frac{\mathrm{d} S_{\mathrm{uf}}(t)}{\mathrm{d} t} \leq\left(1-p_{1}+\frac{\theta}{\mu_{\mathrm{f}}}\right) \Pi_{\mathrm{f}}-$ $\left(\mu_{\mathrm{f}}+\theta\right) S_{\mathrm{uf}}(t), \frac{\mathrm{d} S_{\mathrm{vf}}(t)}{\mathrm{d} t} \leq p_{1} \Pi_{\mathrm{f}}-\left(\theta+\mu_{\mathrm{f}}\right) S_{\mathrm{vf}}(t)$, $\frac{\mathrm{d} S_{\mathrm{um}}(t)}{\mathrm{d} t} \leq\left(1-p_{2}+\frac{\theta}{\mu_{\mathrm{m}}}\right) \Pi_{\mathrm{m}}-\left(\mu_{\mathrm{m}}+\theta\right) S_{\mathrm{um}}(t)$, and $\frac{\mathrm{d} S_{\mathrm{vm}}(t)}{\mathrm{d} t} \leq p_{2} \Pi_{\mathrm{m}}-\left(\theta+\mu_{\mathrm{m}}\right) S_{\mathrm{vm}}(t)$. Then we consider the following comparison system with pulse 


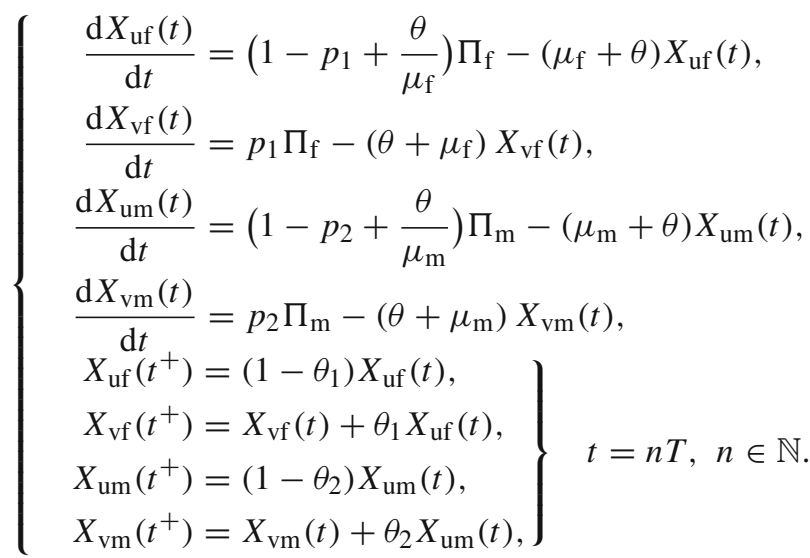

By Lemma 2.2, the periodic solution of system Eq. (17) is given as

$$
\begin{aligned}
& \bar{X}_{\mathrm{ufe}}(t)=\frac{\Pi_{\mathrm{f}}\left(\left(1-p_{1}\right) \mu_{\mathrm{f}}+\theta\right)}{\mu_{\mathrm{f}}\left(\mu_{\mathrm{f}}+\theta\right)} \\
& +\left(S_{\mathrm{uf} 0}-\frac{\Pi_{\mathrm{f}}\left(\left(1-p_{1}\right) \mu_{\mathrm{f}}+\theta\right)}{\mu_{\mathrm{f}}\left(\mu_{\mathrm{f}}+\theta\right)}\right) e^{-\left(\mu_{\mathrm{f}}+\theta\right)(t-n T)}, \\
& \bar{X}_{\mathrm{vf}}(t)=\frac{p_{1} \Pi_{\mathrm{f}}}{\mu_{\mathrm{f}}+\theta} \\
& \quad+\left(S_{\mathrm{vf} 0}-\frac{p_{1} \Pi}{\mu_{\mathrm{f}}+\theta}\right) e^{-\left(\mu_{\mathrm{f}}+\theta\right)(t-n T)}, \\
& \quad \bar{X}_{\mathrm{ume}}(t)=\frac{\Pi_{\mathrm{m}}\left(\left(1-p_{2}\right) \mu_{\mathrm{m}}+\theta\right)}{\mu_{\mathrm{m}}\left(\mu_{\mathrm{m}}+\theta\right)} \\
& \quad+\left(S_{\mathrm{um} 0}-\frac{\Pi_{\mathrm{m}}\left(\left(1-p_{2}\right) \mu_{\mathrm{m}}+\theta\right)}{\mu_{\mathrm{m}}\left(\mu_{\mathrm{m}}+\theta\right)}\right) e^{-\left(\mu_{\mathrm{m}}+\theta\right)(t-n T)}, \\
& \quad n T<t \leq(n+1) T, \\
& \quad \bar{X}_{\mathrm{vm}}(t)=\frac{p_{2} \Pi}{\mu_{\mathrm{m}}+\theta} \\
& \quad\left(S_{\mathrm{vm} 0}-\frac{p_{2} \Pi}{\mu_{\mathrm{m}}+\theta}\right) e^{-\left(\mu_{\mathrm{m}}+\theta\right)(t-n T)},
\end{aligned}
$$

which is globally asymptotically stable, where $S_{\text {uf0, }}$, $S_{\mathrm{vf} 0}, S_{\mathrm{um} 0}$, and $S_{\mathrm{vm} 0}$ are defined in Eq. (14).

Let $\quad\left(S_{\mathrm{uf}}(t), I_{\mathrm{f}}(t), S_{\mathrm{vf}}(t), N_{\mathrm{f}}(t), S_{\mathrm{um}}(t), I_{\mathrm{m}}(t)\right.$, $\left.S_{\mathrm{vm}}(t), N_{\mathrm{m}}(t)\right)$ be the solution of system Eq. (4) with an initial condition Eq. (5) and $S_{\mathrm{uf}}\left(0^{+}\right)=S_{\mathrm{uf} 0}>$ $0, S_{\mathrm{vf}}\left(0^{+}\right)=S_{\mathrm{vf} 0}>0, S_{\mathrm{um}}\left(0^{+}\right)=S_{\mathrm{um} 0}>0$, $S_{\mathrm{vm}}\left(0^{+}\right)=S_{\mathrm{vm} 0}>0,\left(X_{\mathrm{uf}}(t), X_{\mathrm{vf}}(t), X_{\mathrm{um}}(t), X_{\mathrm{vm}}(t)\right)$ be the solution of system Eq. (17) with initial value $X_{\text {uf }}\left(0^{+}\right)=S_{\text {uf } 0}, X_{\mathrm{vf}}\left(0^{+}\right)=S_{\mathrm{vf} 0}, X_{\mathrm{um}}\left(0^{+}\right)=S_{\mathrm{um} 0}$, and $X_{\mathrm{vm}}\left(0^{+}\right)=S_{\mathrm{vm} 0}$. By the comparison theorem for impulsive differential equations [15,32], there exists a constant integer $n_{1}>0$ such that

$$
\begin{gathered}
S_{\mathrm{uf}}(t) \leq X_{\mathrm{uf}}(t)<\bar{X}_{\mathrm{ufe}}(t)+\epsilon, \\
S_{\mathrm{vf}}(t) \leq X_{\mathrm{vf}}(t)<\bar{X}_{\mathrm{vfe}}(t)+\epsilon, \\
S_{\mathrm{um}}(t) \leq X_{\mathrm{vf}}(t)<\bar{X}_{\mathrm{ume}}(t)+\epsilon, \\
S_{\mathrm{vm}}(t) \leq X_{\mathrm{vm}}(t)<\bar{X}_{\mathrm{vme}}(t)+\epsilon,
\end{gathered}
$$

that is

$$
\begin{aligned}
& S_{\mathrm{uf}}(t)<\bar{X}_{\mathrm{ufe}}(t)+\epsilon \\
& \quad \leq \frac{\Pi_{\mathrm{f}}\left(\left(1-p_{1}\right) \mu_{\mathrm{f}}+\theta\right)\left(1-e^{-\left(\mu_{\mathrm{f}}+\theta\right) T}\right)}{\mu_{\mathrm{f}}\left(\mu_{\mathrm{f}}+\theta\right)\left(1-\left(1-\theta_{1}\right) e^{-\left(\mu_{\mathrm{f}}+\theta\right) T}\right)}+\epsilon, \\
& S_{\mathrm{vf}}(t)<\bar{X}_{\mathrm{vfe}}(t)+\epsilon \\
& \quad \leq \frac{p_{1} \Pi_{\mathrm{f}}}{\mu_{\mathrm{f}}+\theta}+\theta_{1} \frac{\Pi_{\mathrm{f}}\left(\left(1-p_{1}\right) \mu_{\mathrm{f}}+\theta\right) e^{-\left(\theta+\mu_{\mathrm{f}}\right) T}}{\mu_{\mathrm{f}}\left(\mu_{\mathrm{f}}+\theta\right)\left(1-\left(1-\theta_{1}\right) e^{-\left(\mu_{\mathrm{f}}+\theta\right) T}\right)} \\
& \quad+\epsilon, \quad n T<t \leq(n+1) T, n>n_{1}, \\
& S_{\mathrm{um}}(t)<\bar{X}_{\mathrm{ume}}(t)+\epsilon \\
& \quad \leq \frac{\Pi_{\mathrm{m}}\left(\left(1-p_{2}\right) \mu_{\mathrm{m}}+\theta\right)\left(1-e^{-\left(\mu_{\mathrm{m}}+\theta\right) T}\right)}{\mu_{\mathrm{m}}\left(\mu_{\mathrm{m}}+\theta\right)\left(1-\left(1-\theta_{2}\right) e^{-\left(\mu_{\mathrm{m}}+\theta\right) T}\right)}+\epsilon, \\
& S_{\mathrm{vm}}(t)<\bar{X}_{\mathrm{vme}}(t)+\epsilon \\
& \quad \leq \frac{p_{2} \Pi}{\mu_{\mathrm{m}}+\theta}+\theta_{2} \frac{\Pi_{\mathrm{m}}\left(\left(1-p_{2}\right) \mu_{\mathrm{m}}+\theta\right) e^{-\left(\theta+\mu_{\mathrm{m}}\right) T}}{\mu_{\mathrm{m}}\left(\mu_{\mathrm{m}}+\theta\right)\left(1-\left(1-\theta_{2}\right) e^{-\left(\mu_{\mathrm{m}}+\theta\right) T}\right)}+\epsilon .
\end{aligned}
$$

Moreover, from the second and sixth equations of system Eq. (4) and from the inequities Eq. (20), we have

$$
\begin{aligned}
& \frac{\mathrm{d}\left(I_{\mathrm{f}}(t)+I_{\mathrm{m}}(t)\right)}{\mathrm{d} t} \\
& \quad=\left(S_{\mathrm{uf}}+\left(1-\Psi_{1}\right) S_{\mathrm{vf}}(t)\right) \beta_{\mathrm{m}} I_{\mathrm{m}}(t) \\
& \quad-\left(\mu_{\mathrm{f}}+\gamma_{\mathrm{f}}+d_{\mathrm{f}}\right) I_{\mathrm{f}}(t) \\
& \quad+\left(S_{\mathrm{um}}(t)+\left(1-\Psi_{2}\right) S_{\mathrm{vm}}(t)\right) \beta_{\mathrm{f}} I_{\mathrm{f}}(t) \\
& \quad-\left(\mu_{\mathrm{m}}+\gamma_{\mathrm{m}}+d_{\mathrm{m}}\right) I_{\mathrm{m}}(t) \\
& \quad \leq\left(S_{\mathrm{uf}}(t)+\left(1-\Psi_{1}\right) S_{\mathrm{vf}}(t)\right) \beta_{\mathrm{m}}\left(I_{\mathrm{m}}(t)+I_{\mathrm{f}}(t)\right)
\end{aligned}
$$




$$
\begin{aligned}
& -\left(S_{\mathrm{um}}(t)+\left(1-\Psi_{2}\right) S_{\mathrm{vm}}(t)\right) \beta_{\mathrm{f}}\left(I_{\mathrm{m}}(t)+I_{\mathrm{f}}(t)\right) \\
& -M^{*}\left(I_{\mathrm{m}}(t)+I_{\mathrm{f}}(t)\right) \\
\leq & {\left[\beta_{\mathrm{m}}\left(\bar{S}_{\mathrm{ufe}}(t)+\left(1-\Psi_{1}\right) \bar{S}_{\mathrm{vfe}}(t)\right)+\beta_{\mathrm{f}}\left(\bar{S}_{\mathrm{ume}}(t)\right.\right.} \\
& \left.+\left(1-\Psi_{2}\right) \bar{S}_{\mathrm{vme}}(t)\right)+\left(\left(2-\Psi_{1}\right) \beta_{\mathrm{m}}+\left(2-\Psi_{2}\right) \beta_{\mathrm{f}}\right) \epsilon \\
& \left.-M^{*}\right]\left(I_{\mathrm{m}}(t)+I_{\mathrm{f}}(t)\right) .
\end{aligned}
$$

By Eq. (3), we can obtain $\lim _{t \rightarrow \infty}\left(I_{\mathrm{f}}(t)+I_{\mathrm{m}}(t)\right)=0$, that is,

$$
\lim _{t \rightarrow \infty} I_{\mathrm{f}}(t)=\lim _{t \rightarrow \infty} I_{\mathrm{m}}(t)=0,
$$

since $I_{\mathrm{f}}(t) \geq 0$ and $I_{\mathrm{m}}(t) \geq 0$. That is, for any $\epsilon_{0}>0$ (sufficiently small), there exists an integer $n_{2}>n_{1}$, such that $I_{\mathrm{f}}(t)<\epsilon_{0}$ and $I_{\mathrm{m}}(t)<\epsilon_{0}$ for all $t>n_{2} T$. From the fourth and eighth equations of system Eq. (4), we also get

$$
\begin{aligned}
& \frac{\mathrm{d} N_{\mathrm{f}}(t)}{\mathrm{d} t} \geq \Pi_{\mathrm{f}}-\mu_{\mathrm{f}} N_{\mathrm{f}}(t)-d_{\mathrm{f}} \epsilon_{0} \\
& \text { and } \frac{\mathrm{d} N_{\mathrm{m}}(t)}{\mathrm{d} t} \geq \Pi_{\mathrm{m}}-\mu_{\mathrm{m}} N_{\mathrm{m}}(t)-d_{\mathrm{m}} \epsilon_{0},
\end{aligned}
$$

for all $t>n_{2} T$. Introduce $Z_{\mathrm{f}}(t)$ and $Z_{\mathrm{m}}(t)$, respectively, as solutions for the ordinary differential equations

$$
\begin{aligned}
& \frac{\mathrm{d} Z_{\mathrm{f}}(t)}{\mathrm{d} t}=\left(\Pi_{\mathrm{f}}-d_{\mathrm{f}} \epsilon_{0}\right)-\mu_{\mathrm{f}} Z_{\mathrm{f}}(t) \text { and } \frac{\mathrm{d} Z_{\mathrm{m}}(t)}{\mathrm{d} t} \\
& \quad=\left(\Pi_{\mathrm{m}}-d_{\mathrm{m}} \epsilon_{0}\right)-\mu_{\mathrm{m}} Z_{n}(t) .
\end{aligned}
$$

Since the equations in Eq. (3) are linear, it is easy to solve them using integrating factor technique and find the the explicit solutions for $Z_{\mathrm{f}}(t)$ and $Z_{\mathrm{m}}(t)$. Consequently, the explicit solutions are

$$
\begin{aligned}
& Z_{\mathrm{f}}(t)=\frac{\left(\Pi_{\mathrm{f}}-d_{\mathrm{f}} \epsilon_{0}\right)}{e^{\mu_{\mathrm{f}} t}}\left[\frac{e^{\mu_{\mathrm{f}} t}-1}{\mu_{\mathrm{f}}}\right] \\
& \text { and } Z_{\mathrm{m}}(t)=\frac{\left(\Pi_{\mathrm{m}}-d_{\mathrm{m}} \epsilon_{0}\right)}{e^{\mu_{\mathrm{m}} t}}\left[\frac{e^{\mu_{\mathrm{m}} t}-1}{\mu_{\mathrm{m}}}\right] .
\end{aligned}
$$

It is clear that $\lim _{t \rightarrow \infty} Z_{\mathrm{f}}(t)=\frac{\Pi_{\mathrm{f}}-d_{\mathrm{f}} \epsilon_{0}}{\mu_{\mathrm{f}}}$ and $\lim _{t \rightarrow \infty} Z_{\mathrm{m}}(t)=\frac{\Pi_{\mathrm{m}}-d_{\mathrm{m}} \epsilon_{0}}{\mu_{\mathrm{m}}}$. Using the comparison theorem for differential equations [33,34], there exists an integer $n_{3}>n_{2}$ such that for all $t>n_{3} T$,

$$
\begin{aligned}
& N_{\mathrm{f}}(t) \geq \frac{\Pi_{\mathrm{f}}-d_{\mathrm{f}} \epsilon_{0}}{\mu_{\mathrm{f}}}-\epsilon_{0} \text { and } N_{\mathrm{m}}(t) \\
& \geq \frac{\Pi_{\mathrm{m}}-d_{\mathrm{m}} \epsilon_{0}}{\mu_{\mathrm{f}}}-\epsilon_{0} .
\end{aligned}
$$

Since $\epsilon_{0}$ is arbitrarily small, from $\lim \sup _{t \rightarrow \infty} N_{\mathrm{f}}(t) \leq$ $\frac{\Pi_{\mathrm{f}}}{\mu_{\mathrm{f}}}, \lim \sup _{t \rightarrow \infty} N_{\mathrm{m}}(t) \leq \frac{\Pi_{\mathrm{m}}}{\mu_{\mathrm{m}}}$, and Eq. (25) we obtain

$\lim _{t \rightarrow \infty} N_{\mathrm{f}}(t)=\frac{\Pi_{\mathrm{f}}}{\mu_{\mathrm{f}}}$ and $\lim _{t \rightarrow \infty} N_{\mathrm{m}}(t)=\frac{\Pi_{\mathrm{m}}}{\mu_{\mathrm{m}}}$.

From Eqs. (21) and (26), there exists $n_{4}>n_{3}$ such that

$$
\begin{aligned}
& I_{\mathrm{f}}(t)<\epsilon_{0}, \quad N_{\mathrm{f}}(t)> \\
& \quad \frac{\Pi_{\mathrm{f}}}{\mu_{\mathrm{f}}}-\epsilon_{0}, \quad I_{\mathrm{m}}(t)<\epsilon_{0}, N_{\mathrm{m}}(t)> \\
& \quad \frac{\Pi_{\mathrm{m}}}{\mu_{\mathrm{m}}}-\epsilon_{0} \text { for } t>n_{4} T .
\end{aligned}
$$

From Eq. (27), the first equation of system Eq. (4) yields

$$
\begin{aligned}
\frac{\mathrm{d}}{S_{\mathrm{uf}}(t)} & =\left(1-p_{1}\right) \Pi_{\mathrm{f}}+\gamma_{\mathrm{f}} I_{\mathrm{f}}(t) \\
& +\theta S_{\mathrm{vf}}(t)-\left(\mu_{\mathrm{f}}+\beta_{\mathrm{m}} I_{m}\right) S_{\mathrm{uf}}(t) \\
= & \left(1-p_{1}\right) \Pi_{\mathrm{f}}+\gamma_{\mathrm{f}} I_{\mathrm{f}}(t)+\theta\left(N_{\mathrm{f}}(t)-I_{\mathrm{f}}(t)-S_{\mathrm{uf}}(t)\right) \\
& -\left(\mu_{\mathrm{f}}+\beta_{\mathrm{m}} I_{m}\right) S_{\mathrm{uf}}(t) \\
= & \left(1-p_{1}\right) \Pi_{\mathrm{f}}-\left(\theta-\gamma_{\mathrm{f}}\right) I_{\mathrm{f}}(t)+\theta\left(N_{\mathrm{f}}(t)-S_{\mathrm{uf}}(t)\right) \\
& -\left(\mu_{\mathrm{f}}+\beta_{\mathrm{m}} I_{m}\right) S_{\mathrm{uf}}(t) \\
> & \left(1-p_{1}\right) \Pi_{\mathrm{f}}-\left(\theta-\gamma_{\mathrm{f}}\right) \epsilon_{0}+\theta\left(\frac{\Pi_{\mathrm{f}}}{\mu_{\mathrm{f}}}-\epsilon_{0}\right) \\
& -\left(\theta+\mu_{\mathrm{f}}+\beta_{\mathrm{m}} \epsilon_{0}\right) S_{\mathrm{uf}}(t) \\
= & \left(\left(1-p_{1}\right) \Pi_{\mathrm{f}}+\frac{\theta \Pi_{\mathrm{f}}}{\mu_{\mathrm{f}}}\right. \\
& \left.+\epsilon_{0}\left(\gamma_{\mathrm{f}}-2 \theta\right)\right)-\left(\theta+\mu_{\mathrm{f}}+\beta_{\mathrm{m}} \epsilon_{0}\right) S_{\mathrm{uf}}(t)
\end{aligned}
$$

Moreover, the second equation of system Eq. (4) yields

$$
\begin{gathered}
\frac{\mathrm{d} S_{\mathrm{vf}}(t)}{\mathrm{d} t}=p_{1} \Pi_{\mathrm{f}}-\left(\theta+\mu_{\mathrm{f}}+\left(1-\Psi_{1}\right) \beta_{\mathrm{m}} I_{\mathrm{m}}(t)\right) S_{\mathrm{vf}}(t) \\
\geq p_{1} \Pi_{\mathrm{f}}-\left(\theta+\mu_{\mathrm{f}}+\left(1-\Psi_{1}\right) \beta_{\mathrm{m}} \epsilon_{0}\right) S_{\mathrm{vf}}(t) .
\end{gathered}
$$

In a similar way, from the fourth and sixth equations of system Eq. (4), we obtain

$$
\begin{aligned}
& \frac{\mathrm{d} S_{\mathrm{um}}(t)}{\mathrm{d} t}>\left(\left(1-p_{2}\right) \Pi_{\mathrm{m}}+\frac{\theta \Pi_{\mathrm{m}}}{\mu_{\mathrm{m}}}+\epsilon_{0}\left(\gamma_{\mathrm{f}}-2 \theta\right)\right) \\
& \quad-\left(\theta+\mu_{\mathrm{m}}+\beta_{\mathrm{f}} \epsilon_{0}\right) S_{\mathrm{um}}(t), \text { and } \\
& \frac{\mathrm{d} S_{\mathrm{vm}}(t)}{\mathrm{d} t}>p_{2} \Pi_{\mathrm{m}}-\left(\theta+\mu_{\mathrm{m}}+\left(1-\Psi_{2}\right) \beta_{\mathrm{f}} \epsilon_{0}\right) S_{\mathrm{vm}}(t) .
\end{aligned}
$$

Consider the following comparison differential equations for all $t>n_{5} T$ and $n>n_{5}$, 


$$
\left.\begin{array}{rl}
\frac{\mathrm{d} Y_{\mathrm{uf}}(t)}{\mathrm{d} t} & =\left(\left(1-p_{1}\right) \Pi_{\mathrm{f}}+\frac{\theta \Pi_{\mathrm{f}}}{\mu_{\mathrm{f}}}+\epsilon_{0}\left(\gamma_{\mathrm{f}}-2 \theta\right)\right)-\left(\theta+\mu_{\mathrm{f}}+\beta_{\mathrm{m}} \epsilon_{0}\right) Y_{\mathrm{uf}}(t), \\
\frac{\mathrm{d} Y_{\mathrm{vf}}(t)}{\mathrm{d} t} & =p_{1} \Pi_{\mathrm{f}}-\left(\theta+\mu_{\mathrm{f}}+\left(1-\Psi_{1}\right) \beta_{\mathrm{m}} \epsilon_{0}\right) Y_{\mathrm{vf}}(t), \\
\frac{\mathrm{d} Y_{\mathrm{um}}(t)}{\mathrm{d} t} & =\left(\left(1-p_{2}\right) \Pi_{\mathrm{m}}+\frac{\theta \Pi_{\mathrm{m}}}{\mu_{\mathrm{m}}}+\epsilon_{0}\left(\gamma_{\mathrm{m}}-2 \theta\right)\right)-\left(\theta+\mu_{\mathrm{m}}+\beta_{\mathrm{f}} \epsilon_{0}\right) Y_{\mathrm{um}}(t), \\
\frac{\mathrm{d} Y_{\mathrm{vm}}(t)}{\mathrm{d} t} & =p_{2} \Pi_{\mathrm{m}}-\left(\theta+\mu_{\mathrm{m}}+\left(1-\Psi_{2}\right) \beta_{\mathrm{f}} \epsilon_{0}\right) Y_{\mathrm{vm}}(t), \\
Y_{\mathrm{uf}}\left(t^{+}\right) & =\left(1-\theta_{1}\right) Y_{\mathrm{uf}}(t), \\
Y_{\mathrm{vf}}\left(t^{+}\right) & =Y_{\mathrm{vf}}(t)+\theta_{1} Y_{\mathrm{uf}}(t), \\
Y_{\mathrm{um}}\left(t^{+}\right) & =\left(1-\theta_{2}\right) X_{\mathrm{um}}(t), \\
Y_{\mathrm{vm}}\left(t^{+}\right) & =Y_{\mathrm{vm}}(t)+\theta_{2} Y_{\mathrm{um}}(t),
\end{array}\right\} \quad t=n T, n \in \mathbb{N} .
$$

Using Lemma 2.2, the unique periodic solution of system Eq. (29), which is globally asymptotically stable, is

$$
\begin{aligned}
& \bar{Y}_{\mathrm{ufe}}(t)=\pi_{1}+\left(Y_{\mathrm{uf} 0}-\pi_{1}\right) e^{-\left(\theta+\mu_{\mathrm{f}}+\beta_{\mathrm{m}} \epsilon_{0}\right)(t-n T)}, \\
& n T<t \leq(n+1) T, \\
& \bar{Y}_{\mathrm{vf}}(t)=\frac{p_{1} \Pi_{\mathrm{f}}}{\theta+\mu_{\mathrm{f}}+\left(1-\Psi_{1}\right) \beta_{\mathrm{m}} \epsilon_{0}} \\
& \quad+\left(Y_{\mathrm{vf} 0}-\frac{p_{1} \Pi}{\theta+\mu_{\mathrm{f}}+\left(1-\Psi_{1}\right) \beta_{\mathrm{m}} \epsilon_{0}}\right)
\end{aligned}
$$

$$
\begin{aligned}
& e^{-\left(\theta+\mu_{\mathrm{f}}+\left(1-\Psi_{1}\right) \beta_{\mathrm{m}} \epsilon_{0}\right)(t-n T)}, \\
& \bar{Y}_{\mathrm{ume}}(t)=\pi_{2}+\left(Y_{\mathrm{um} 0}-\pi_{2}\right) e^{-\left(\theta+\mu_{\mathrm{m}}+\beta_{\mathrm{f}} \epsilon_{0}\right)(t-n T)} \\
& n T<t \leq(n+1) T \\
& \bar{Y}_{\mathrm{vm}}(t)=\frac{p_{2} \Pi}{\theta+\mu_{\mathrm{m}}+\left(1-\Psi_{2}\right) \beta_{\mathrm{f}} \epsilon_{0}} \\
& \quad+\left(Y_{\mathrm{vm} 0}-\frac{p_{2} \Pi}{\theta+\mu_{\mathrm{m}}+\left(1-\Psi_{2}\right) \beta_{\mathrm{f}} \epsilon_{0}}\right) \\
& \quad e^{-\left(\theta+\mu_{\mathrm{m}}+\left(1-\Psi_{2}\right) \beta_{\mathrm{f}} \epsilon_{0}\right)(t-n T)}
\end{aligned}
$$

where

$$
\begin{aligned}
& \pi_{1}=\frac{\left(\left(1-p_{1}\right) \Pi_{\mathrm{f}}+\frac{\theta \Pi_{\mathrm{f}}}{\mu_{\mathrm{f}}}+\epsilon_{0}\left(\gamma_{\mathrm{f}}-2 \theta\right)\right)}{\theta+\mu_{\mathrm{f}}+\beta_{\mathrm{m}} \epsilon_{0}}, \\
& Y_{\mathrm{uf} 0}=\pi_{1} \frac{\left(1-\theta_{1}\right)\left(1-e^{-\left(\theta+\mu_{\mathrm{f}}+\beta_{\mathrm{m}} \epsilon_{0}\right) T}\right)}{\left(1-\left(1-\theta_{1}\right) e^{-\left(\theta+\mu_{\mathrm{f}}+\beta_{\mathrm{m}} \epsilon_{0}\right) T}\right)}, \\
& Y_{\mathrm{vf} 0}=\frac{p_{1} \Pi_{\mathrm{f}}}{\theta+\mu_{\mathrm{f}}+\left(1-\Psi_{1}\right) \beta_{\mathrm{m}} \epsilon_{0}}+\theta_{1} \frac{\Pi_{\mathrm{f}}\left(\left(1-p_{1}\right) \mu_{\mathrm{f}}+\theta\right)}{\left(\mu_{\mathrm{f}}\left(\theta+\mu_{\mathrm{f}}+\left(1-\Psi_{1}\right) \beta_{\mathrm{m}} \epsilon_{0}\right)\right)\left(1-\left(1-\theta_{1}\right) e^{-\left(\theta+\mu_{\mathrm{f}}+\left(1-\Psi_{1}\right) \beta_{\mathrm{m}} \epsilon_{0}\right) T}\right)}, \\
& \pi_{2}=\frac{\left(\left(1-p_{2}\right) \Pi_{\mathrm{m}}+\frac{\theta \Pi_{\mathrm{m}}}{\mu_{\mathrm{m}}}+\epsilon_{0}\left(\gamma_{\mathrm{m}}-2 \theta\right)\right)}{\theta+\mu_{\mathrm{m}}+\beta_{\mathrm{f}} \epsilon_{0}}, \\
& Y_{\mathrm{um} 0}=\pi_{2} \frac{\left(1-\theta_{2}\right)\left(1-e^{-\left(\theta+\mu_{\mathrm{m}}+\beta_{\mathrm{f}} \epsilon_{0}\right) T}\right)}{\left(1-\left(1-\theta_{2}\right) e^{-\left(\theta+\mu_{\mathrm{m}}+\beta_{\mathrm{f}} \epsilon_{0}\right) T}\right)}, \\
& Y_{\mathrm{vm} 0}=\frac{\Pi_{\mathrm{m}}\left(\left(1-p_{2}\right) \mu_{\mathrm{m}}+\theta\right)}{\theta+\mu_{\mathrm{m}}+\left(1-\Psi_{2}\right) \beta_{\mathrm{f}} \epsilon_{0}}+\theta_{2} \frac{p_{2}}{\left(\mu_{\mathrm{m}}\left(\theta+\mu_{\mathrm{m}}+\left(1-\Psi_{2}\right) \beta_{\mathrm{f}} \epsilon_{0}\right)\right)\left(1-\left(1-\theta_{2}\right) e^{-\left(\theta+\mu_{\mathrm{m}}+\left(1-\Psi_{2}\right) \beta_{\mathrm{f}} \epsilon_{0}\right) T}\right)} .
\end{aligned}
$$


Based on the comparison theorem for impulsive differential equations, there exists an integer $n_{6}>n_{5}$ such that

$$
\begin{aligned}
S_{\mathrm{uf}}(t) & >\bar{Y}_{\mathrm{ufe}}(t)-\epsilon_{0}, \\
S_{\mathrm{vf}}(t) & >\bar{Y}_{\mathrm{vfe}}(t)-\epsilon_{0}, \\
S_{\mathrm{um}}(t) & >\bar{Y}_{\mathrm{ume}}(t)-\epsilon_{0}, \quad n T<t \leq(n+1) T, \quad n>n_{6}, \\
S_{\mathrm{vm}}(t) & >\bar{Y}_{\mathrm{vme}}(t)-\epsilon_{0} .
\end{aligned}
$$

Since $\epsilon$ and $\epsilon_{0}$ are assumed to be arbitrarily small, it follows from Eqs. (20) and (31) that

$$
\begin{aligned}
\lim _{t \rightarrow \infty} S_{\mathrm{uf}}(t)=\lim _{t \rightarrow \infty} \bar{S}_{\mathrm{ufe}}(t), & \\
& \lim _{t \rightarrow \infty} S_{\mathrm{vf}}(t)=\lim _{t \rightarrow \infty} \bar{S}_{\mathrm{vfe}}(t), \quad \lim _{t \rightarrow \infty} S_{\mathrm{um}}(t) \\
= & \lim _{t \rightarrow \infty} \bar{S}_{\mathrm{ume}}(t), \quad \lim _{t \rightarrow \infty} S_{\mathrm{vm}}(t)=\lim _{t \rightarrow \infty} \bar{S}_{\mathrm{vme}}(t) .
\end{aligned}
$$

Therefore by Eqs. (21) and (32) the disease-free periodic solution $\left(\bar{S}_{\mathrm{ufe}}(t), 0, \bar{S}_{\mathrm{vfe}}(t), \bar{S}_{\mathrm{ume}}(t), 0, \bar{S}_{\mathrm{vme}}(t)\right)$ is globally asymptotically stable. This completes the proof.

The physical interpretation of Theorem 3.1 is that if the pulse reproduction number is less than unity the disease will vanish from the population.

Next, we find the critical proportion of the female susceptible vaccination, that is, the $\theta_{1}=\theta_{1}^{*}$ such that $R_{0}\left(\theta_{1}^{*}\right)=1$. To easily manipulate the equation we assume that the pulse vaccination for males is not applied. Let $X_{1}=\frac{\Pi_{\mathrm{f}}\left(\left(1-p_{1}\right) \mu_{\mathrm{f}}+\theta\right)}{\mu_{\mathrm{f}}\left(\mu_{\mathrm{f}}+\theta\right)}$ and further if $\theta_{2}=0$ we get the reproduction number for males from evaluating the integral Eq. (15) that $R_{\mathrm{om}}=$ $\frac{\beta_{\mathrm{f}}}{M^{*}}\left[\frac{\Pi_{\mathrm{m}}\left(\left(1-p_{2}\right) \mu_{\mathrm{m}}+\theta\right)}{\mu_{\mathrm{m}}\left(\mu_{\mathrm{m}}+\theta\right)}+\frac{\left(1-\Psi_{2}\right) p_{2} \Pi_{\mathrm{m}}}{\mu_{\mathrm{m}}+\theta}\right]$. We can easily get that
Corollary 3.1 The disease-free periodic solution $\left(\bar{S}_{\mathrm{ufe}}(t), 0, \bar{S}_{\mathrm{vfe}}(t), \bar{S}_{\mathrm{ume}}(t), 0, \bar{S}_{\mathrm{vme}}(t)\right)$ of system Eq. (4) is globally attractive provided that $\theta_{1}>\theta_{1}^{*}$ where $\theta_{1}^{*}$ is given by Eq. (33).

The epidemiological interpretation of Corollary 3.1 is that if the pulse vaccination $\theta_{1}$ is greater than the critical vaccination $\theta_{1}^{*}$ the disease will vanish from the population.

\section{The uniform persistence of the HPV model}

Within this section, the theory of the uniform persistence of the disease is well described and analyzed. We say the disease is persistent if the infected population continues for sufficiently long periods above a certain positive point.

Definition 4.1 System Eq. (4) is uniformly persistent if there is $\eta>0$ (independent of the initial data) so that each solution $\left(S_{\mathrm{uf}}(t), I_{\mathrm{f}}(t), S_{\mathrm{vf}}(t), N_{\mathrm{f}}(t), S_{\mathrm{um}}(t)\right.$, $\left.I_{\mathrm{m}}(t), S_{\mathrm{vm}}(t), N_{\mathrm{m}}(t)\right)$ with the initial conditions Eq. (5) satisfies

$$
\begin{aligned}
& \liminf _{t \rightarrow \infty} S_{\mathrm{uf}}(t) \geq \eta, \quad \liminf _{t \rightarrow \infty} I_{\mathrm{f}}(t) \geq \eta, \\
& \liminf _{t \rightarrow \infty} S_{\mathrm{vf}}(t) \geq \eta, \quad \liminf _{t \rightarrow \infty} S_{\mathrm{um}}(t) \geq \eta, \\
& \liminf _{t \rightarrow \infty} I_{\mathrm{m}}(t) \geq \eta, \quad \liminf _{t \rightarrow \infty} S_{\mathrm{vm}}(t) \geq \eta, \\
& \liminf _{t \rightarrow \infty} N_{\mathrm{f}}(t) \geq \eta, \quad \liminf _{t \rightarrow \infty} N_{\mathrm{m}}(t) \geq \eta .
\end{aligned}
$$

For any $r_{0}>0$ and $t>0$, we define

$$
\begin{aligned}
& H\left(r_{0}, t\right)=\left(\left(\bar{S}_{\mathrm{ufe}}(t)+\left(1-\Psi_{1}\right) \bar{S}_{\mathrm{vfe}}(t)\right)\right. \\
& \left.\quad \beta_{\mathrm{m}} r_{0}-\left(\mu_{\mathrm{f}}+\gamma_{\mathrm{f}}+d_{\mathrm{f}}\right)\right) \\
& -\left(\left(\bar{S}_{\mathrm{ume}}(t)+\left(1-\Psi_{2}\right) \bar{S}_{\mathrm{vme}}(t)\right) \frac{\beta_{\mathrm{f}}}{r_{0}}\right. \\
& \left.-\left(\mu_{\mathrm{m}}+\gamma_{\mathrm{m}}+d_{\mathrm{m}}\right)\right)
\end{aligned}
$$

$\theta_{1}^{*}=\frac{\left[\frac{\mu_{\mathrm{f}}+\theta}{1-e^{-\left(\mu_{\mathrm{m}}+\theta\right) T}}\left(\left(1-R_{\mathrm{mo}}\right) \frac{M^{*} T}{\beta_{\mathrm{m}}}-X_{1} T-\left(1-\Psi_{1}\right) \frac{p_{1} \Pi_{\mathrm{f}}}{\mu_{\mathrm{f}}+\theta}\right)\right]\left(1-e^{-\left(\mu_{\mathrm{f}}+\theta\right) T}\right)}{-X_{1} \Psi_{1}-e^{-\left(\mu_{\mathrm{f}}+\theta\right) T}\left[\frac{\mu_{\mathrm{f}}+\theta}{1-e^{-\left(\mu_{\mathrm{m}}+\theta\right) T}}\left(\left(1-R_{\mathrm{mo}}\right) \frac{M^{*} T}{\beta_{\mathrm{m}}}-X_{1} T-\left(1-\Psi_{1}\right) \frac{p_{1} \Pi_{\mathrm{f}}}{\mu_{\mathrm{f}}+\theta}\right)\right]}$.
Furthermore, from Eq. (15), we have

$$
\frac{\mathrm{d} R_{0}}{\mathrm{~d} \theta_{1}}<0 \text {. }
$$

Therefore, $R_{0}<0$ only when $\theta_{1}>\theta_{1}^{*}$. We have the following corollary, which is a direct consequence of Theorem 3.1. and

$F\left(r_{0}, t\right)=r_{0} I_{\mathrm{f}}(t)-I_{\mathrm{m}}(t)$,

where $\bar{S}_{\text {ufe }}(t), \bar{S}_{\text {vfe }}(t), \bar{S}_{\text {ume }}(t)$, and $\bar{S}_{\text {vme }}$ are given by Eq. (3) and $I_{\mathrm{f}}(t)$ and $I_{\mathrm{m}}(t)$ are elements of the solutions of system Eq. (4). 
Theorem 4.1 [23] If there exist positive constants $r_{0}$ and $\alpha$ such that

$$
\begin{aligned}
& R_{1}=\int_{0}^{T}\left(\left(\bar{S}_{\mathrm{ufe}}(\tau)+\left(1-\Psi_{1}\right) \bar{S}_{\mathrm{vfe}}(\tau)\right)\right. \\
& \left.\beta_{\mathrm{m}} r_{0}-\left(\mu_{\mathrm{f}}+\gamma_{\mathrm{f}}+d_{\mathrm{f}}\right)\right) \mathrm{d} \tau \\
& R_{2}=\int_{0}^{T}\left(\left(\bar{S}_{\mathrm{ume}}(\tau)+\left(1-\Psi_{2}\right) \bar{S}_{\mathrm{vme}}(\tau)\right)\right. \\
& \left.\quad \frac{\beta_{\mathrm{f}}}{r_{0}}-\left(\mu_{\mathrm{m}}+\gamma_{\mathrm{m}}+d_{\mathrm{m}}\right)\right) \mathrm{d} \tau,
\end{aligned}
$$

and $H\left(r_{0}, t\right)<0$ for all $t \geq 0$, then system Eq. (4) is uniformly persistent.

Proof Since $H\left(r_{0}, t\right)<0$, it is possible to choose a positive constant $\alpha$ such that $H\left(r_{0}, t\right)<-\alpha$. Moreover, by Eqs. (35) and (36), we can choose very small positive constants $\epsilon, \epsilon_{1}, \eta_{1}, \eta_{2}$, and $\eta_{3}$ such that

$$
\begin{aligned}
& \int_{0}^{T}\left(\left(\left(\bar{S}_{\mathrm{ume}}(\tau)-\epsilon\right)+\left(1-\Psi_{2}\right)\left(\bar{S}_{\mathrm{vme}}(\tau)-\epsilon\right)\right)\right. \\
& \left.\quad \frac{\beta_{\mathrm{f}}}{r_{0}}-\left(\mu_{\mathrm{m}}+\gamma_{\mathrm{m}}+d_{\mathrm{m}}\right)\right) \mathrm{d} \tau>\eta_{1} \\
& \int_{0}^{T}\left(\left(\left(\bar{S}_{\mathrm{ufe}}(\tau)-\epsilon\right)+\left(1-\Psi_{1}\right)\left(\bar{S}_{\mathrm{vfe}}(\tau)-\epsilon\right)\right)\right. \\
& \left.\beta_{\mathrm{m}} r_{0}-\left(\mu_{\mathrm{f}}+\gamma_{\mathrm{f}}+d_{\mathrm{f}}\right)\right) \mathrm{d} \tau>\eta_{2} \\
& \int_{0}^{T}\left(\left(\left(\bar{S}_{\mathrm{ume}}(\tau)+\epsilon\right)+\left(1-\Psi_{2}\right)\left(\bar{S}_{\mathrm{vme}}(\tau)+\epsilon\right)\right)\right. \\
& \left.\beta_{\mathrm{f}} \epsilon-\left(\mu_{\mathrm{m}}+\gamma_{\mathrm{m}}+d_{\mathrm{m}}\right) \epsilon_{1}\right) \mathrm{d} \tau<-\eta_{3}
\end{aligned}
$$

and

$$
\begin{aligned}
& H\left(r_{0}, t\right)=\left(\left(\left(\bar{S}_{\mathrm{ufe}}(t)\right)+\epsilon\right)\right. \\
& \left.\left.\quad+\left(1-\Psi_{1}\right)\left(\bar{S}_{\mathrm{vfe}}(t)+\epsilon\right)\right) \beta_{\mathrm{m}} r_{0}-\left(\mu_{\mathrm{f}}+\gamma_{\mathrm{f}}+d_{\mathrm{f}}\right)\right) \\
& \quad-\left(\left(\left(\bar{S}_{\mathrm{ume}}(t)-\epsilon\right)+\left(1-\Psi_{2}\right)\left(\bar{S}_{\mathrm{vme}}(t)-\epsilon\right)\right) \quad(40)\right. \\
& \left.\quad \frac{\beta_{\mathrm{f}}}{r_{0}}-\left(\mu_{\mathrm{m}}+\gamma_{\mathrm{m}}+d_{\mathrm{m}}\right)\right)<0 .
\end{aligned}
$$

First, we prove that

$$
\limsup _{t \rightarrow \infty} I_{\mathrm{f}}(t) \geq \epsilon
$$

If Eq. (41) was not true, then there would be a positive integer $n_{1}$ such that $0<I_{\mathrm{f}}(t)<\epsilon$ for all $t \geq n_{1} T$. If $I_{\mathrm{m}}(t) \geq \epsilon_{1}$ for all $t \geq n_{1} T$, then from the sixth equation of system Eq. (4) and the fact $0<I_{\mathrm{f}}(t)<\epsilon$ we obtain

$$
\begin{aligned}
& I_{\mathrm{m}}(t)=I_{\mathrm{m}}\left(n_{1} T\right)+\int_{n_{1} T}^{t}\left(\left(\bar{S}_{\mathrm{ume}}(s)+\left(1-\Psi_{2}\right) \bar{S}_{\mathrm{vme}}(s)\right)\right. \\
& \left.\quad \beta_{\mathrm{f}} I_{f}(s)-\left(\mu_{\mathrm{m}}+\gamma_{\mathrm{m}}+d_{\mathrm{m}}\right) I_{\mathrm{m}}(s)\right) \mathrm{d} s \\
& \leq I_{\mathrm{m}}\left(n_{1} T\right)+\int_{n_{1} T}^{t}\left(\left(\left(\bar{S}_{\mathrm{ume}}(s)+\epsilon\right)\right.\right. \\
& \left.\quad+\left(1-\Psi_{2}\right)\left(\bar{S}_{\mathrm{vme}}(s)+\epsilon\right)\right) \\
& \left.\quad \beta_{\mathrm{f}} \epsilon-\left(\mu_{\mathrm{m}}+\gamma_{\mathrm{m}}+d_{\mathrm{m}}\right) \epsilon_{1}\right) \mathrm{d} s
\end{aligned}
$$

for all $t \geq n_{1} T$. From Eq. (39), it directly follows that $\lim _{t \rightarrow \infty} I_{\mathrm{m}}(t)=-\infty$. This is a contradiction to the positivity of $I_{\mathrm{m}}(t)$. Therefore, there exists $t_{1} \geq n_{1} T$ such that $I_{\mathrm{m}}\left(t_{1}\right) \leq \epsilon_{1}$. Next, we claim

$$
I_{\mathrm{m}}(t)<\epsilon_{1}+2 \beta_{\mathrm{f}} \epsilon\left(\frac{\Pi_{\mathrm{m}}}{\mu_{\mathrm{m}}}+\epsilon\right) T
$$

for all $t \geq t_{1}$. If this fails, there exists $t_{2}>t_{1}$ such that $I_{\mathrm{m}}\left(t_{2}\right)>\epsilon_{1}+2 \beta_{\mathrm{f}} \epsilon\left(\frac{\Pi_{\mathrm{m}}}{\mu_{\mathrm{m}}}+\epsilon\right) T$. Then there must exist $t_{3} \in\left(t_{1}, t_{2}\right)$ such that $I_{\mathrm{m}}\left(t_{3}\right)=\epsilon_{1}$ and $I_{\mathrm{m}}(t)>\epsilon_{1}$ for $t \in\left(t_{3}, t_{2}\right)$. We can choose a positive integer $m$ such that $t_{2} \in\left(t_{3}+m T, t_{3}+(m+1) T\right)$. Integrating the fifth equation of model Eq. (4) from $t_{3}$ to $t_{2}$ and using Eq. (39), we obtain

$$
\begin{aligned}
\epsilon_{1}+ & 2 \beta_{\mathrm{f}} \epsilon\left(\frac{\Pi_{\mathrm{m}}}{\mu_{\mathrm{m}}}+\epsilon\right) T \\
< & I_{\mathrm{m}}\left(t_{2}\right)=I_{\mathrm{m}}\left(t_{3}\right)+\int_{t_{3}}^{t_{2}} \\
& \left(\left(\bar{S}_{\mathrm{ume}}(s)+\left(1-\Psi_{2}\right) \bar{S}_{\mathrm{vme}}(s)\right) \beta_{\mathrm{f}} I_{f}(s)\right. \\
& \left.-\left(\mu_{\mathrm{m}}+\gamma_{\mathrm{m}}+d_{\mathrm{m}}\right) I_{\mathrm{m}}(s)\right) \mathrm{d} s \\
\leq & \epsilon_{1}+\left(\int_{t_{3}}^{t_{3}+m T}+\int_{t_{3}+m T}^{t_{2}}\right) \\
& \left(\left(\left(\bar{S}_{\mathrm{ume}}(s)+\epsilon\right)+\left(1-\Psi_{2}\right)\left(\bar{S}_{\mathrm{vme}}(s)+\epsilon\right)\right) \beta_{\mathrm{f}} \epsilon\right. \\
& \left.-\left(\mu_{\mathrm{m}}+\gamma_{\mathrm{m}}+d_{\mathrm{m}}\right) \epsilon_{1}\right) \mathrm{d} s \\
< & \epsilon_{1}+2 \beta_{\mathrm{f}} \epsilon\left(\frac{\Pi_{\mathrm{m}}}{\mu_{\mathrm{m}}}+\epsilon\right) T .
\end{aligned}
$$

This is a contradiction. Thus, Eq. (42) is valid. 
It follows from the first and the third equations of system Eq. (4) that

$$
\begin{aligned}
& \frac{\mathrm{d} S_{\mathrm{uf}}(t)}{\mathrm{d} t}=\left(1-p_{1}\right) \Pi_{\mathrm{f}}+\gamma_{\mathrm{f}} I_{\mathrm{f}}(t)+\theta S_{\mathrm{vf}}(t) \\
& \quad-\left(\mu_{\mathrm{f}}+\beta_{\mathrm{m}} I_{m}^{*}(t)\right) S_{\mathrm{uf}}(t) \\
& \geq\left(1-p_{1}\right) \Pi_{\mathrm{f}}-\frac{\beta_{\mathrm{m}} \Pi_{\mathrm{f}}}{\mu_{\mathrm{f}}} \\
& \quad\left(\epsilon_{1}+2 \beta_{\mathrm{f}} \epsilon\left(\frac{\Pi_{\mathrm{m}}}{\mu_{\mathrm{m}}}+\epsilon\right) T\right)-\mu_{\mathrm{f}} S_{\mathrm{uf}}(t), \\
& \quad \frac{\mathrm{d} S_{\mathrm{vf}}(t)}{\mathrm{d} t}=p_{1} \Pi_{\mathrm{f}}-\left(\theta+\mu_{\mathrm{f}}+\left(1-\Psi_{1}\right) \beta_{\mathrm{m}} I_{m}^{*}(t)\right) \\
& S_{\mathrm{vf}}(t) \geq p_{1} \Pi_{\mathrm{f}}-\frac{\left(1-\Psi_{1}\right) \beta_{\mathrm{m}} \Pi_{\mathrm{f}}}{\mu_{\mathrm{f}}} \\
& \left(\epsilon_{1}+2 \beta_{\mathrm{f}} \epsilon\left(\frac{\Pi_{\mathrm{m}}}{\mu_{\mathrm{m}}}+\epsilon\right) T\right)-\left(\theta+\mu_{\mathrm{f}}\right) S_{\mathrm{vf}}(t) .
\end{aligned}
$$

Consider the following comparison impulsive differential equations for $t \geq t_{0}$,
By Eq. (43) and the comparison theorem for impulsive differential equations, there exists an integer $n_{2} \geq n_{1}$ such that

$$
S_{\mathrm{uf}}(t)>\bar{u}_{1 f}(t)-\epsilon_{1}, \quad S_{\mathrm{vf}}(t)>\bar{v}_{1 f}(t)-\epsilon_{1} .
$$

Moreover, by Eq. (19) and the comparison theorem for impulsive differential equation, there exists an integer $n_{3} \geq n_{2}$ such that

$$
S_{\mathrm{uf}}(t)<\bar{S}_{\mathrm{ufe}}(t)+\epsilon, \quad S_{\mathrm{vf}}(t)<\bar{S}_{\mathrm{vfe}}(t)+\epsilon,
$$

for all $t \in(n T,(n+1) T]$ and $n \geq n_{3}$. Since $\epsilon$ and $\epsilon_{1}$ are small enough, from Eqs. (46) and (47), we have

$$
\begin{gathered}
\bar{S}_{\mathrm{ufe}}(t)-\epsilon_{1}<S_{\mathrm{uf}}(t)<\bar{S}_{\mathrm{ufe}}(t)+\epsilon, \\
\bar{S}_{\mathrm{vfe}}(t)-\epsilon_{1}<S_{\mathrm{vf}}(t)<\bar{S}_{\mathrm{vfe}}(t)+\epsilon .
\end{gathered}
$$

Furthermore, we prove that there exists a positive number $n_{5} \geq n_{4}$ such that

$F\left(r_{0}, t\right) \leq 0$ for all $t \geq n_{5} T$.

$$
\begin{aligned}
& \left.\begin{array}{l}
\frac{\mathrm{d} u_{1 f}(t)}{\mathrm{d} t}=\left(1-p_{1}\right) \Pi_{\mathrm{f}}-\frac{\beta_{\mathrm{m}} \Pi_{\mathrm{f}}}{\mu_{\mathrm{f}}}\left(\epsilon_{1}+2 \beta_{\mathrm{f}} \epsilon\left(\frac{\Pi_{\mathrm{m}}}{\mu_{\mathrm{m}}}+\epsilon\right) T\right)-\mu_{\mathrm{f}} u_{1 f}(t), \\
\frac{\mathrm{d} v_{1 f}(t)}{\mathrm{d} t}=p_{1} \Pi_{\mathrm{f}}-\frac{\left(1-\Psi_{1}\right) \beta_{\mathrm{m}} \Pi_{\mathrm{f}}}{\mu_{\mathrm{f}}}\left(\epsilon_{1}+2 \beta_{\mathrm{f}} \epsilon\left(\frac{\Pi_{\mathrm{m}}}{\mu_{\mathrm{m}}}+\epsilon\right) T\right)-\left(\theta+\mu_{\mathrm{f}}\right) v_{1 f}(t),
\end{array}\right\} t \neq n T, n \in \mathbb{N} \\
& u_{1 f}\left(t^{+}\right)=\left(1-\theta_{1}\right) u_{1 f}(t) \text {, } \\
& \left.v_{1 f}\left(t^{+}\right)=v_{1 f}(t)+\theta_{1} u_{1 f}(t),\right\} \\
& t=n T, n \in \mathbb{N} \text {. }
\end{aligned}
$$

In view of Lemma 2.2, the unique periodic solution for system Eq. (44) is

$$
\begin{aligned}
\bar{u}_{1 f}(t)= & \frac{\left(1-p_{1}\right) \Pi_{\mathrm{f}}-\frac{\beta_{\mathrm{m}} \Pi_{\mathrm{f}}}{\mu_{\mathrm{f}}}\left(\epsilon_{1}+2 \beta_{\mathrm{f}} \epsilon\left(\frac{\Pi_{\mathrm{m}}}{\mu_{\mathrm{m}}}+\epsilon\right) T\right)}{\mu_{\mathrm{f}}} \\
& +\left(u_{1 f}^{*}-\frac{\left(1-p_{1}\right) \Pi_{\mathrm{f}}-\frac{\beta_{\mathrm{m}} \Pi_{\mathrm{f}}}{\mu_{\mathrm{f}}}\left(\epsilon_{1}+2 \beta_{\mathrm{f}} \epsilon\left(\frac{\Pi_{\mathrm{m}}}{\mu_{\mathrm{m}}}+\epsilon\right) T\right)}{\mu_{\mathrm{f}}}\right) e^{-\mu_{\mathrm{f}}(t-n T)}, \\
\bar{v}_{1 f}(t)= & \frac{p_{1} \Pi_{\mathrm{f}}-\frac{\left(1-\Psi_{1}\right) \beta_{\mathrm{m}} \Pi_{\mathrm{f}}}{\mu_{\mathrm{f}}}\left(\epsilon_{1}+2 \beta_{\mathrm{f}} \epsilon\left(\frac{\Pi_{\mathrm{m}}}{\mu_{\mathrm{m}}}+\epsilon\right) T\right)}{\theta+\mu_{\mathrm{f}}} \\
& +\frac{\theta_{1}}{1-e^{-\left(\theta+\mu_{\mathrm{f}}\right) T}} \frac{\left(1-p_{1}\right) \Pi_{\mathrm{f}}}{\mu_{\mathrm{f}}} \frac{1-e^{-\mu_{\mathrm{f}} T}}{1-\left(1-\theta_{1}\right) e^{-\mu_{\mathrm{f}} T}} e^{-\left(\theta+\mu_{\mathrm{f}}\right)(t-n T)},
\end{aligned}
$$

which is globally stable, where

$$
u_{1 f}^{*}=\frac{\left(1-p_{1}\right) \Pi_{\mathrm{f}}-\frac{\beta_{\mathrm{m}} \Pi_{\mathrm{f}}}{\mu_{\mathrm{f}}}\left(\epsilon_{1}+2 \beta_{\mathrm{f}} \epsilon\left(\frac{\Pi_{\mathrm{m}}}{\mu_{\mathrm{m}}}+\epsilon\right) T\right)}{\mu_{\mathrm{f}}} \frac{\left(1-\theta_{1}\right)\left(1-e^{-\mu_{\mathrm{f}} T}\right)}{1-\left(1-\theta_{1}\right) e^{-\mu_{\mathrm{f}} T}} .
$$


If this fails, there exist two cases:

(i) $F\left(r_{0}, t\right)>0$ for all $t \geq n_{4} T$ and

(ii) $F\left(r_{0}, t\right)$ oscillates around 0 for all $t$ large enough.

If case $(i)$ holds, then from the fifth equation of system Eq. (4), we obtain

$$
\begin{aligned}
& \frac{\mathrm{d} I_{\mathrm{m}}(t)}{\mathrm{d} t}>\left(S_{\mathrm{um}}(t)+\left(1-\Psi_{2}\right) S_{\mathrm{vm}}(t)\right) \\
& \frac{\beta_{\mathrm{f}} I_{\mathrm{m}}(t)}{r_{0}}-\left(\mu_{\mathrm{m}}+\gamma_{\mathrm{m}}+d_{\mathrm{m}}\right) I_{\mathrm{m}}(t) \\
& >\left(\left(\left(S_{\mathrm{um}}(t)-\epsilon\right)+\left(1-\Psi_{2}\right)\left(S_{\mathrm{vm}}(t)-\epsilon\right)\right)\right. \\
& \left.\frac{\beta_{\mathrm{f}}}{r_{0}}-\left(\mu_{\mathrm{m}}+\gamma_{\mathrm{m}}+d_{\mathrm{m}}\right)\right) I_{\mathrm{m}}(t) .
\end{aligned}
$$

Integrating this inequality from $n_{4} T$ to $t$, we obtain

$$
\begin{aligned}
I_{\mathrm{m}}(t) & >I_{\mathrm{m}}(n T) \exp \left\{\int _ { n _ { 4 } T } ^ { t } \left[\left(\left(S_{\mathrm{um}}(\tau)-\epsilon\right)\right.\right.\right. \\
& \left.\left.\left.+\left(1-\Psi_{2}\right)\left(S_{\mathrm{vm}}(\tau)-\epsilon\right)\right) \frac{\beta_{\mathrm{f}}}{r_{0}}-\left(\mu_{\mathrm{m}}+\gamma_{\mathrm{m}}+d_{\mathrm{m}}\right)\right] \mathrm{d} \tau\right\} .
\end{aligned}
$$

From this and Eq. (37), we obtain that $\lim _{t \rightarrow \infty} I_{\mathrm{m}}(t)=$ $\infty$. This contradicts the boundedness of $I_{\mathrm{m}}(t)$. Therefore, case $(i)$ is incorrect.

Next, we claim that case (ii) is also incorrect. Suppose there exist two time sequences $t_{\mathrm{m}}$ and $s_{\mathrm{m}}$ satisfying

$n_{4} T<s_{1}<t_{1}<s_{2}<t_{2}<\cdots<s_{\mathrm{m}}<t_{\mathrm{m}}<\cdots$

and $\lim _{m \rightarrow \infty} s_{\mathrm{m}}=\infty$ such that $F\left(r_{0}, s_{\mathrm{m}}\right)=0$, $d F\left(r_{0}, s_{\mathrm{m}}\right) / \mathrm{d} s_{\mathrm{m}} \geq 0, F\left(r_{0}, s_{\mathrm{m}}\right)>0$ for all $t \in$ $\bigcup_{m=1}^{\infty}\left(s_{\mathrm{m}}, t_{\mathrm{m}}\right)$, and $F\left(r_{0}, s_{\mathrm{m}}\right) \leq 0$ for all $t \notin$ $\bigcup_{m=1}^{\infty}\left(s_{\mathrm{m}}, t_{\mathrm{m}}\right)$. Consequently, we have $r_{0} I_{\mathrm{f}}\left(s_{\mathrm{m}}\right)=$ $I_{\mathrm{m}}\left(s_{\mathrm{m}}\right)$ and

$$
\begin{aligned}
\frac{\mathrm{d} F\left(r_{0}, s_{\mathrm{m}}\right)}{\mathrm{d} s_{\mathrm{m}}}= & r_{0} \frac{\mathrm{d} I_{\mathrm{f}}\left(s_{\mathrm{m}}\right)}{\mathrm{d} s_{\mathrm{m}}}-\frac{\mathrm{d} I_{\mathrm{m}}\left(s_{\mathrm{m}}\right)}{\mathrm{d} s_{\mathrm{m}}} \\
= & I_{\mathrm{m}}\left(s_{\mathrm{m}}\right)\left[\left(\left(\bar{S}_{\text {ufe }}(t)+\left(1-\Psi_{1}\right) \bar{S}_{\text {vfe }}(t)\right) \beta_{\mathrm{m}} r_{0}-\left(\mu_{\mathrm{f}}+\gamma_{\mathrm{f}}+d_{\mathrm{f}}\right)\right)\right. \\
& \left.-\left(\left(\bar{S}_{\text {ume }}(t)+\left(1-\Psi_{2}\right) \bar{S}_{\text {vme }}(t)\right) \frac{\beta_{\mathrm{f}}}{r_{0}}-\left(\mu_{\mathrm{m}}+\gamma_{\mathrm{m}}+d_{\mathrm{m}}\right)\right)\right] \\
\geq & 0 .
\end{aligned}
$$

This is in contraction with Eq. (40). Moreover, by the above discussion, Eq. (49) is valid.

From the second equation of system Eq. (4) and using Eqs. (40) and (48), we have

$$
\begin{aligned}
\frac{\mathrm{d} I_{\mathrm{f}}(t)}{\mathrm{d} t}= & \left(S_{\mathrm{uf}}(t)+\left(1-\Psi_{1}\right) S_{\mathrm{vf}}(t)\right) \beta_{\mathrm{m}} I_{\mathrm{m}}(t) \\
& -\left(\mu_{\mathrm{f}}+\gamma_{\mathrm{f}}+d_{\mathrm{f}}\right) I_{\mathrm{f}} \\
\geq & {\left[\left(S_{\mathrm{uf}}(t)-\epsilon\right)+\left(1-\Psi_{2}\right)\left(S_{\mathrm{vf}}(t)-\epsilon\right)\right) \beta_{\mathrm{m}} r_{0} }
\end{aligned}
$$

$$
\left.-\left(\mu_{\mathrm{f}}+\gamma_{\mathrm{f}}+d_{\mathrm{f}}\right)\right] I_{\mathrm{f}}(t) .
$$

Integrating this inequality from $n_{5} T$ to $t$, it follows that

$$
\begin{aligned}
I_{\mathrm{f}}(t) & \geq I_{\mathrm{f}}\left(n_{5} T\right) \exp \left\{\int _ { n _ { 5 } T } ^ { t } \left[\left(\left(S_{\mathrm{uf}}(t)-\epsilon\right)\right.\right.\right. \\
& \left.\left.\left.+\left(1-\Psi_{2}\right)\left(S_{\mathrm{vf}}(t)-\epsilon\right)\right) \beta_{\mathrm{m}} r_{0}-\left(\mu_{\mathrm{f}}+\gamma_{\mathrm{f}}+d_{\mathrm{f}}\right)\right] \mathrm{d} \tau\right\} .
\end{aligned}
$$

It is easy to obtain that $\lim _{t \rightarrow \infty} I_{\mathrm{f}}(t)=\infty$ from Eq. (38). This is a contradiction to the boundedness of $I_{\mathrm{f}}(t)$. Therefore, Eq. (41) is correct.

Finally, we prove that

$\liminf _{t \rightarrow \infty} I_{\mathrm{f}}(t) \geq \epsilon$

for any solution $\left(S_{\mathrm{uf}}(t), I_{\mathrm{f}}(t), S_{\mathrm{vf}}(t), S_{\mathrm{um}}(t), I_{\mathrm{m}}(t)\right.$, $\left.S_{\mathrm{vm}}(t)\right)$ of system Eq. (4) with initial value Eq. (5). By Eq. (38), it is possible to choose two positive constants $\omega$ and $k$ such that

$$
\begin{aligned}
& \int_{t}^{t+\xi}\left[\left(S_{\mathrm{uf}}(\tau)-\epsilon\right)+\left(1-\Psi_{2}\right)\left(S_{\mathrm{vf}}(\tau)-\epsilon\right)\right) \\
& \left.\quad \beta_{\mathrm{m}} r_{0}-\left(\mu_{\mathrm{f}}+\gamma_{\mathrm{f}}+d_{\mathrm{f}}\right)\right] \mathrm{d} \tau>k
\end{aligned}
$$

for all $t \geq n_{5} T$ and $\xi>\omega$. If Eq. (50) was not true, then there would exist a sequence of initial values $Y_{n}=$ $\left(S_{\mathrm{uf} n}, I_{f n}, S_{\mathrm{vf} n}, S_{\mathrm{um} n}, I_{m n}, S_{\mathrm{vm} n}\right) \in \mathrm{R}^{6}(n=1,2, \ldots)$ such that

$$
\liminf _{t \rightarrow \infty} I_{\mathrm{f}}\left(t, Y_{n}\right) \geq \frac{\epsilon}{n^{2}} \text {. }
$$

By Eq. (41), for every $n$, there exist two time sequences $t_{j}^{(n)}$ and $s_{j}^{(n)}$ satisfying

$0<s_{1}^{(n)}<t_{1}^{(n)}<s_{2}^{(n)}<t_{2}^{(n)}<\cdots<s_{j}^{(n)}<t_{j}^{(n)}<\cdots$ and $\lim _{j \rightarrow \infty} s_{j}^{(n)}=\infty$ such that

$I_{\mathrm{f}}\left(s_{j}^{n}, Y_{n}\right)=\frac{\epsilon}{n}, \quad I_{\mathrm{f}}\left(t_{j}^{n}, Y_{n}\right)=\frac{\epsilon}{n^{2}}$,

and

$\frac{\epsilon}{n^{2}}<I_{\mathrm{f}}\left(t, Y_{n}\right)<\frac{\epsilon}{n}$ for all $t \in\left(s_{j}^{(n)}, t_{j}^{(n)}\right)$.

From the second equation of system Eq. (4), we get that

$$
\begin{aligned}
& \frac{\mathrm{d} I_{\mathrm{f}}\left(t, Y_{n}\right)}{\mathrm{d} t}=\left(S_{\mathrm{uf}}(t)+\left(1-\Psi_{1}\right) S_{\mathrm{vf}}(t)\right) \beta_{\mathrm{m}} I_{\mathrm{m}}(t) \\
& \quad-\left(\mu_{\mathrm{f}}+\gamma_{\mathrm{f}}+d_{\mathrm{f}}\right) I_{\mathrm{f}}(t) \geq-\left(\mu_{\mathrm{f}}+\gamma_{\mathrm{f}}+d_{\mathrm{f}}\right) I_{\mathrm{f}}\left(t, Y_{n}\right) .
\end{aligned}
$$

It follows by integrating this inequality from $s_{j}^{(n)}$ to $t_{j}^{(n)}$ that

$$
I_{\mathrm{f}}\left(t_{j}^{n}, Y_{n}\right) \geq I_{\mathrm{f}}\left(s_{j}^{n}, Y_{n}\right) \exp \left\{-\left(\mu_{\mathrm{f}}+\gamma_{\mathrm{f}}+d_{\mathrm{f}}\right)\left(t_{j}^{n}-s_{j}^{n}\right)\right\} .
$$


Therefore, by Eq. (53), we obtain

$\frac{\epsilon}{n^{2}} \geq \frac{\epsilon}{n} \exp \left\{-\left(\mu_{\mathrm{f}}+\gamma_{\mathrm{f}}+d_{\mathrm{f}}\right)\left(t_{j}^{n}-s_{j}^{n}\right)\right\}$,

that is,

$t_{j}^{n}-s_{j}^{n} \geq \frac{\ln n}{\mu_{\mathrm{f}}+\gamma_{\mathrm{f}}+d_{\mathrm{f}}} \rightarrow \infty$ as $j \rightarrow \infty$.

For each $n$, from the first equation of system Eq. (4), we obtain

$$
\begin{aligned}
\frac{\mathrm{d} S_{\mathrm{uf}}(t)}{\mathrm{d} t} \geq & \left(1-p_{1}\right) \Pi_{\mathrm{f}}-\frac{\beta_{\mathrm{m}} \Pi_{\mathrm{f}}}{\mu_{\mathrm{f}}}\left(\epsilon_{1}+2 \beta_{\mathrm{f}} \epsilon\left(\frac{\Pi_{\mathrm{m}}}{\mu_{\mathrm{m}}}+\epsilon\right) T\right) \\
& -\mu_{\mathrm{f}} u_{1 f}(t) \text { for all } t \in\left[t_{j}^{n}, s_{j}^{n}\right] .
\end{aligned}
$$

Assume that $\bar{S}_{\mathrm{f}}(t)$ is the solution of the first equation of Eq. (44) with initial value $\bar{S}_{\mathrm{f}}\left(s_{j}^{(n)}\right)=S_{\mathrm{f}}\left(s_{j}^{(n)}, Y_{n}\right)$. Using the comparison theorem of impulsive differential equations, we obtain $S_{\mathrm{uf}}\left(t, Y_{n}\right) \geq \bar{S}_{\mathrm{f}}(t)$. By using Eqs. (46) and (48), there is a constant $\bar{T}>0$, independent of $n$ and $j$, and moreover, there exists a positive constant $T^{*}$, independent of any $n$ and $j$, such that $t_{j}^{(n)}-s_{j}^{(n)}>$ $T^{*}$. We choose $n$ large enough to make $t_{j}^{n}-s_{j}^{n}>$ $T^{*}+\omega$. By Eq. (54), there exists $T_{1} \geq n_{5} T$ such that $F\left(r_{0}, t\right)=r_{0} I_{\mathrm{f}}(t)-I_{\mathrm{m}}(t) \leq 0$ for $t \geq T_{1}$.

Finally, from the second equation of system Eq. (4) and from Eq. (49), we obtain

$$
\begin{aligned}
\frac{\mathrm{d} I_{\mathrm{f}}(t)}{\mathrm{d} t}= & \left(S_{\mathrm{uf}}(t)+\left(1-\Psi_{1}\right) S_{\mathrm{vf}}(t)\right) \beta_{\mathrm{m}} I_{\mathrm{m}}(t) \\
& -\left(\mu_{\mathrm{f}}+\gamma_{\mathrm{f}}+d_{\mathrm{f}}\right) I_{\mathrm{f}} \geq\left[\left(S_{\mathrm{uf}}(t)+\left(1-\Psi_{2}\right)\right.\right. \\
& \left.\left.\times\left(S_{\mathrm{vf}}(t)\right)\right) \beta_{\mathrm{m}} r_{0}-\left(\mu_{\mathrm{f}}+\gamma_{\mathrm{f}}+d_{\mathrm{f}}\right)\right] I_{\mathrm{f}}(t) .
\end{aligned}
$$

It follows by integrating this inequality from $s_{j}^{(n)}+T^{*}$ to $t_{j}^{(n)}$ that

$$
\begin{aligned}
\frac{\epsilon}{n^{2}}= & I_{\mathrm{f}}\left(t_{j}^{(n)}, Y_{n}\right) \geq I_{\mathrm{f}}\left(s_{j}^{(n)}+T^{*}, Y_{n}\right) \\
\geq & \frac{\epsilon}{n^{2}} \exp \left\{\int _ { s _ { j } ^ { ( n ) } + T ^ { * } } ^ { t _ { j } ^ { ( n ) } } \left[\left(S_{\mathrm{uf}}(\tau)-\epsilon\right)+\left(1-\Psi_{2}\right)\right.\right. \\
& \left.\left.\times\left(S_{\mathrm{vf}}(\tau)-\epsilon\right) \beta_{\mathrm{m}} r_{0}-\left(\mu_{\mathrm{f}}+\gamma_{\mathrm{f}}+d_{\mathrm{f}}\right)\right] \mathrm{d} \tau\right\} \\
\geq & \frac{\epsilon}{n^{2}} .
\end{aligned}
$$

This is a contradiction, and so we proved that Eq. (50) is correct. From $F\left(r_{0}, t\right)=r_{0} I_{\mathrm{f}}(t)-I_{\mathrm{m}}(t) \leq 0$ for $t \geq T_{1}$, we obtain

$\liminf _{t \rightarrow \infty} I_{\mathrm{m}}(t) \geq \liminf _{t \rightarrow \infty} r_{0} I_{\mathrm{f}}(t) \geq r_{0} \epsilon$.
From this and Eq. (50), we conclude that the HPV disease system Eq. (4) is uniformly persistent. And therefore, the theorem is proved.

Corollary 4.1 Assume that $\theta_{1}<\theta_{1}^{*}$, then system Eq. (4) is permanent.

\section{Numerical simulation and discussion}

In this section, we investigate some numerical simulations to demonstrate the key theoretical results and the feasibility of impulsive vaccination control strategy for various control parameters using the RungeKutta method in the MATLAB program. The description of the parameters used for simulation are listed in Table 1.

Let the initial values be $\left(S_{\mathrm{uf} 0}, I_{\mathrm{f} 0}, S_{\mathrm{vf} 0}, S_{\mathrm{um} 0}, I_{m o}\right.$, $\left.S_{\mathrm{vm} 0}\right)=(1000,10,0,1000,10,0)$. We set the parameter values $\Pi_{\mathrm{f}}=5, \Pi_{\mathrm{m}}=5, \beta_{\mathrm{f}}=0.004, \beta_{\mathrm{m}}=$ $0.0004, \theta_{1}=\theta_{2}=0.5$ and other parameters are as given in Table 1. The time span is further assumed to be 75 years with vaccination cycle every 5 years. It is found out that $X_{1}=\frac{\Pi_{\mathrm{f}}}{\mu_{\mathrm{f}}\left(\theta+\mu_{\mathrm{f}}\right)}\left(\left(1-p_{1}\right) \mu_{\mathrm{f}}+\right.$ $\theta)=\frac{5}{0.033(0.1+0.033)}((1-0.77) 0.033+0.1)=$ 121.125 and $X_{2}=\frac{\Pi_{\mathrm{m}}}{\mu_{\mathrm{m}}\left(\theta+\mu_{\mathrm{m}}\right)}\left(\left(1-p_{2}\right) \mu_{\mathrm{m}}+\theta\right)=$ $\frac{5}{0.033(0.1+0.033)}((1-0.77) 0.033+0.1)=121.125$. $S_{\text {uf } 0}=\frac{X_{1} \times(1-0.5) \times\left(1-e^{-(0.033+0.1)}\right)}{1-(1-0.5) \times e^{-(0.033+0.1)}}=39.6462, S_{\text {vf } 0}=$ $\frac{0.77 \times 5}{0.033+0.1}+\frac{0.5 \times X_{1}}{1-(1-0.5) \times e^{-(0.033+0.1)}}=110.3538 . S_{\mathrm{um} 0}=$ $\frac{X_{2} \times(1-0.5) \times\left(1-e^{-(0.033+0.1)}\right)}{1-(1-0.5) \times e^{-(0.033+0.1)}}=39.6462, S_{\mathrm{vm} 0}=\frac{0.77 \times 5}{0.033+0.1}$ $+\frac{0.5 \times X_{1}}{1-(1-0.5) \times e^{-(0.033+0.1)}}=110.3538$.

$$
\begin{aligned}
R_{0} & =\frac{0.0004}{(0.033+0.77+0.01) \times 5}(121.125 \times 5 \\
& +\frac{(39.6462-121.125)}{0.033+0.1} \times\left(1-e^{-(0.033+0.1)}\right) \\
& +\frac{0.9}{0.033+0.1}(0.77 \times 5 \times 5 \\
& \left.\left.+\left(110.3538-\frac{0.77 \times 5}{0.033+0.1}\right)\left(1-e^{-(0.033+0.1)}\right)\right)\right) \\
& +\frac{0.004}{(0.033+0.77+0.01) \times 5} \\
& \left(121.125 \times 5+\frac{(39.6462-121.125)}{0.033+0.1} \times\right. \\
& \left(1-e^{-(0.033+0.1)}\right) \\
& +\frac{0.9}{0.033+0.1}(0.77 \times 5 \times 5+
\end{aligned}
$$


Table 1 Description of the biological parameters

\begin{tabular}{|c|c|c|c|}
\hline Parameters & Interpretation & Units & Source \\
\hline$\pi_{\mathrm{f}}$ & Recruitment rate of sexually active female population & 50 males year $^{-1}$ & {$[26]$} \\
\hline$\pi_{\mathrm{m}}$ & Recruitment rate of sexually active male population & 50 females year $^{-1}$ & {$[26]$} \\
\hline$p_{1}$ & Proportion of female children vaccinated & 0.77 & {$[26]$} \\
\hline$p_{2}$ & Proportion of male children vaccinated & 0.77 & {$[26]$} \\
\hline$\beta_{\mathrm{m}}$ & Effective transmission rate of HPV from male to female & Varies & Assumed \\
\hline$\beta_{\mathrm{f}}$ & Effective transmission rate of HPV from female to male & Varies & Assumed \\
\hline$\theta$ & The rate of waning of the vaccine induced immunity & 0.1 year $^{-1}$ & {$[28]$} \\
\hline $1 / \gamma_{\mathrm{f}}$ & Females' infectious period & 1.3 & {$[35]$} \\
\hline $1 / \gamma_{\mathrm{m}}$ & Males' infectious period & 0.6 & {$[36]$} \\
\hline $1 / \mu_{\mathrm{f}}$ & The average duration of the sexual activity period for males & 30 years & {$[28]$} \\
\hline $1 / \mu_{\mathrm{m}}$ & The average duration of the sexual activity period for females & 30 years & {$[28]$} \\
\hline $1-\Psi_{1}$ & Females vaccine efficacy & 0.9 year $^{-1}$ & {$[37]$} \\
\hline $1-\Psi_{2}$ & Males vaccine efficacy & 0.9 year $^{-1}$ & {$[37]$} \\
\hline$d_{\mathrm{f}}$ & Disease induced death rate of females & 0.01 year $^{-1}$ & Assumed \\
\hline$d_{\mathrm{m}}$ & Disease induced death rate of males & 0.0004 year $^{-1}$ & Assumed \\
\hline
\end{tabular}

$$
\begin{aligned}
& \left.\left.\left(110.3538-\frac{0.77 \times 5}{0.033+0.1}\right)\left(1-e^{-(0.033+0.1)}\right)\right)\right) \\
= & 0.7644<1 .
\end{aligned}
$$

The reproduction number is $R_{0}=0.7644$. The evolution of the susceptible females and the phase portrait graph of the infected versus the susceptible females are depicted in Fig. 1. Moreover, the evolution of the susceptible males and the phase portrait graph of the infected versus the susceptible males are depicted in Fig. 2. As it can be seen from the time series of susceptible unvaccinated female and male populations, the system evolves by the standard SIV S model following the implementation of each pulse vaccination until the next pulse is applied at time $t_{n+1}=t_{n}+5$ (Figs. $1 \mathrm{a}, 2 \mathrm{a})$. As a result of the pulse vaccination, the susceptible unvaccinated population is diminishing and shifted to the vaccinated population. The phase portrait of the infectious versus susceptible unvaccinated females and infectious versus susceptible unvaccinated males with impulsive vaccination shows that there is a decline and gradually convergence to a disease-free equilibrium where the solution trajectories ultimately reach the susceptible axes (Figs. 1b, 2b).

Since $R_{0}=0.7644<1$, by Theorem 3.1 , the system produces a global asymptotically stable periodic solution (Fig. 3), that is, the disease will gradually van- ish. Figures 3a, b depict that the disease dies out faster when the pulse vaccination is implemented.

Next, we assume $\beta_{\mathrm{m}}=0.001, \beta_{\mathrm{f}}=0.01$, the condition parameter $r_{0}=0.8$ and other parameters are as in Table 1 . We obtain that $X_{1}=$ $\frac{50}{0.033(0.1+0.033)}((1-0.77) 0.033+0.1)=1211.3$ and $X_{2}=\frac{50}{0.033(0.1+0.033)}((1-0.77) 0.033+0.1)=$ 1211.3. Moreover, $S_{\text {uf } 0}=\frac{X_{1} \times(1-0.5) \times\left(1-e^{-(0.033+0.1)}\right)}{1-(1-0.5) \times e^{-(0.033+0.1)}}=$ $396.4619, S_{\text {vf } 0}=\frac{0.77 \times 5}{0.033+0.1}+\frac{0.5 \times X_{1}}{1-(1-0.5) \times e^{-(0.033+0.1)}}=$ 1103.5,

$S_{\mathrm{um} 0}=\frac{X_{2} \times(1-0.5) \times\left(1-e^{-(0.033+0.1)}\right)}{1-(1-0.5) \times e^{-(0.033+0.1)}}=396.4619$, $S_{\mathrm{vm} 0}=\frac{0.77 \times 5}{0.033+0.1}+\frac{0.5 \times X_{1}}{1-(1-0.5) \times e^{-(0.033+0.1)}}=1103.5$.

$$
\begin{aligned}
R_{1}= & 0.001 \times 0.8[1211.3 \times 5+ \\
& \frac{(396.4619-121.125)}{0.033+0.1} \times\left(1-e^{-(0.033+0.1)}\right) \\
& +\frac{0.9}{0.033+0.1}(0.77 \times 50 \times 5 \\
& \left.\left.+\left(1103.5-\frac{0.77 \times 5}{0.033+0.1}\right)\left(1-e^{-(0.033+0.1)}\right)\right)\right] \\
& -(0.033+0.77+0.01) \times 5 \\
= & 1.5838>0
\end{aligned}
$$




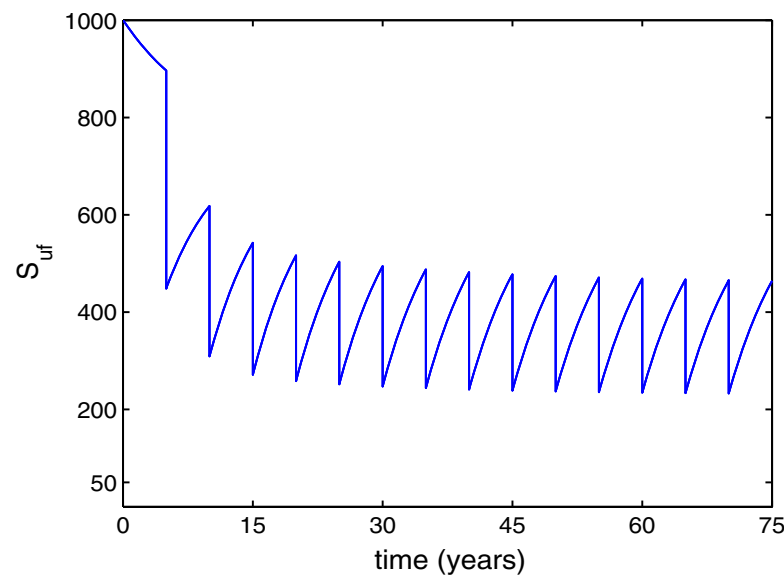

(a) Time series of $S_{u f}$.

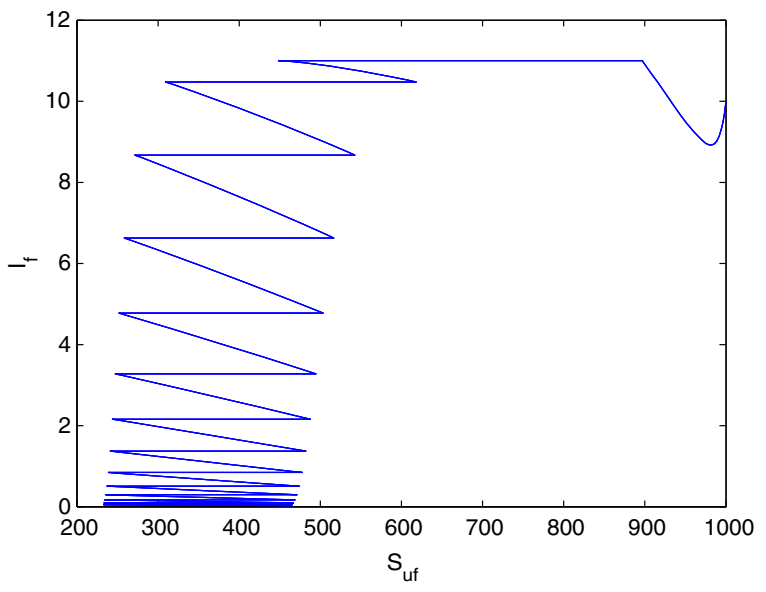

(b) Phase portrait of $I_{f}$ vs $S_{u f}$.

Fig. 1 This figure shows the time series of the susceptible unvaccinated females and the phase portrait of $I_{\mathrm{f}}$ versus $S_{\mathrm{uf}}$ with parameter values $T=5, \Pi_{\mathrm{f}}=5, \Pi_{\mathrm{m}}=5, \beta_{\mathrm{f}}=0.004, \beta_{\mathrm{m}}=0.0004, \theta_{1}=\theta_{2}=0.5$ and other parameters are as in Table 1

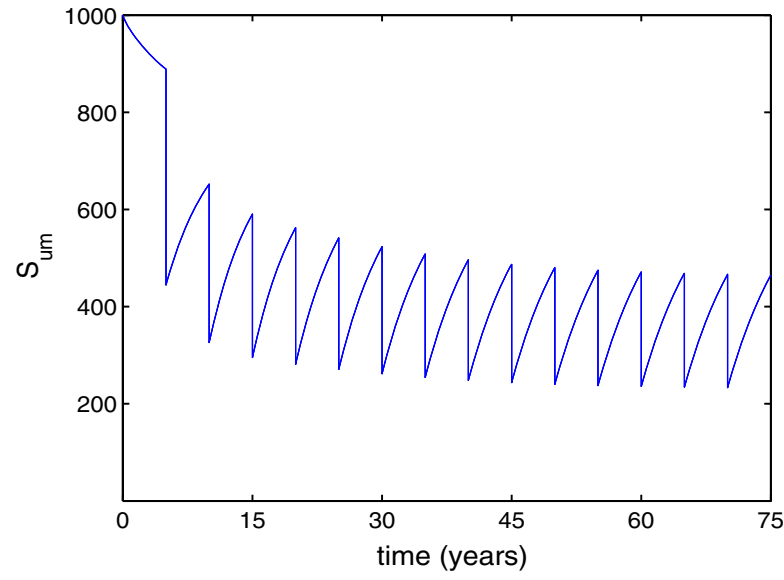

(a) Time series of $S_{u m}$.

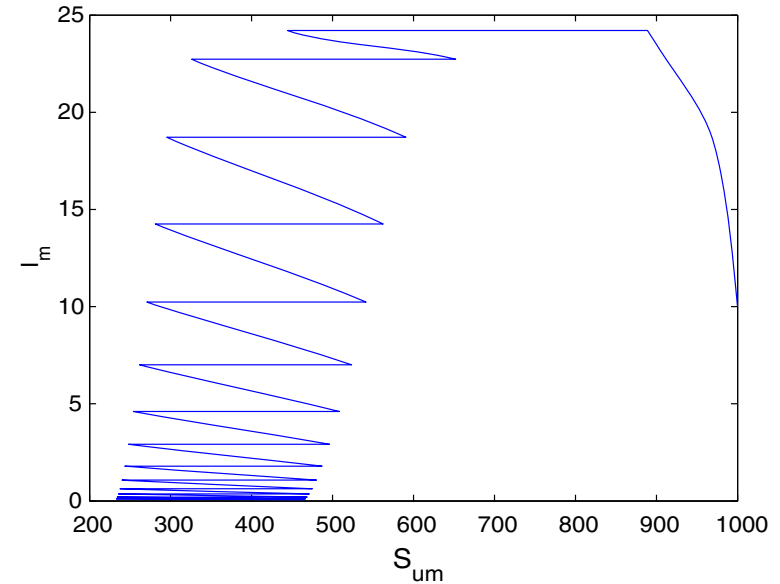

(b) Phase portrait $I_{m}$ vs $S_{u m}$.

Fig. 2 This figure shows the time series of the susceptible unvaccinated males and the phase portrait of $I_{\mathrm{m}}$ versus $S_{\text {um }}$ with parameter values $T=5, \Pi_{\mathrm{f}}=5, \Pi_{\mathrm{m}}=5, \beta_{\mathrm{f}}=0.004, \beta_{\mathrm{m}}=0.0004, \theta_{1}=\theta_{2}=0.5$ and other parameters are as in Table 1

$$
\begin{aligned}
& R_{2}=\frac{0.01}{0.8}[1211.3 \times 5 \\
& +\frac{(396.4619-121.125)}{0.033+0.1} \times\left(1-e^{-(0.033+0.1)}\right) \\
& +\frac{0.9}{0.033+0.1}(0.77 \times 50 \times 5+ \\
& \left.\left.\left(1103.5-\frac{0.77 \times 5}{0.033+0.1}\right)\left(1-e^{-(0.033+0.1)}\right)\right)\right] \\
& -(0.033+1.67+0.001) \times 5 \\
& =79.7235>0
\end{aligned}
$$

with

$$
\begin{aligned}
& H\left(r_{0}, t\right)=0.001 \times 0.8[1211.3+ \\
& (396.4619-121.125) \times\left(1-e^{-(0.033+0.1)}\right) \\
& \quad+0.9(288.7500+(1103.5-288.7500) \\
& \left.\left.\left(1-e^{-(0.033+0.1)}\right)\right)\right]-(0.033+0.77+0.01) \\
& \quad-\left[\frac{0.01}{0.8}[1211.3+(396.4619-121.125) \times\right. \\
& \quad\left(1-e^{-(0.033+0.1)}\right)
\end{aligned}
$$




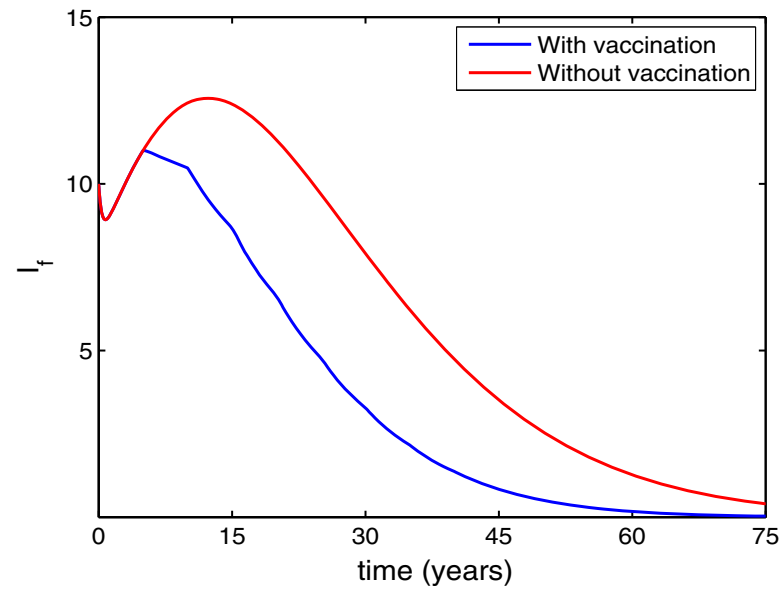

(a) Time series of Infected females.

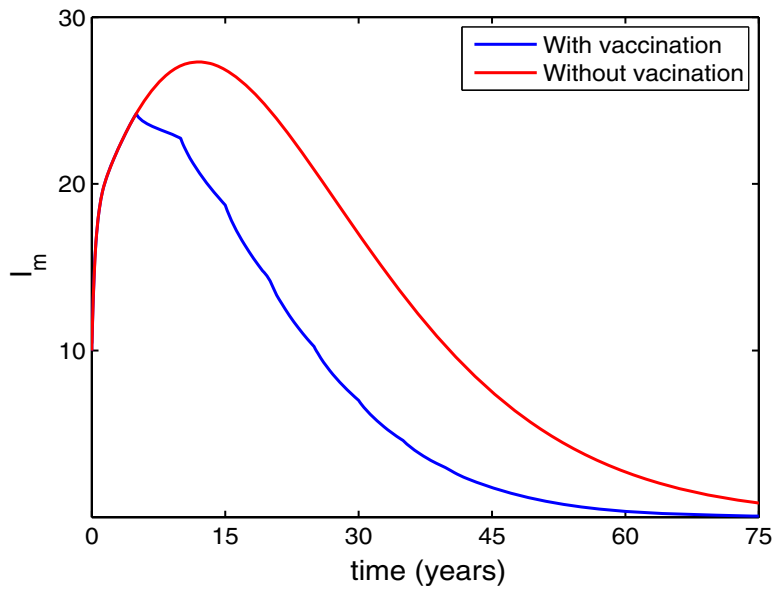

(b) Time series of infected males.

Fig. 3 Time evolution of infected individuals before and after initiation of periodic vaccination with the parameters $T=5$, $\Pi_{\mathrm{f}}=5$, $\Pi_{\mathrm{m}}=5, \beta_{\mathrm{f}}=0.004, \beta_{\mathrm{m}}=0.0004, \theta_{1}=\theta_{2}=0.5$ and other parameters are as in Table 1

$$
\begin{aligned}
& +0.9(288.7500+(1103.5-288.7500) \\
& \left.\left.\left.\left(1-e^{-(0.033+0.1)}\right)\right)\right]-(0.033+1.67+0.001)\right] \\
= & -15.8599<0 .
\end{aligned}
$$

and

$$
\begin{aligned}
R_{0} & =\frac{0.001}{(0.033+0.77+0.01) \times 5}[1211.3 \times 5 \\
& +\frac{(396.4619-121.125)}{0.033+0.1} \times\left(1-e^{-(0.033+0.1)}\right) \\
& +\frac{0.9}{0.033+0.1}(0.77 \times 50 \times 5 \\
& \left.\left.+\left(1103.5-\frac{0.77 \times 5}{0.033+0.1}\right)\left(1-e^{-(0.033+0.1)}\right)\right)\right] \\
& +\frac{0.01}{(0.033+0.77+0.01) \times 5} \\
& {\left[1211.3 \times 5+\frac{(396.4619-121.125)}{0.033+0.1}\right.} \\
& \times\left(1-e^{-(0.033+0.1)}\right) \\
& +\frac{0.9}{0.033+0.1}(0.77 \times 50 \times 5+ \\
& \left(1103.5-\frac{0.77 \times 5}{0.033+0.1}\right) \\
& \left.\left.\left(1-e^{-(0.033+0.1)}\right)\right)\right] \\
= & 19.1101>1 .
\end{aligned}
$$

Obviously, the system Eq. (4) is uniformly persistent and has a positive periodic solution using Theorem 4.1. Biologically the disease will exist in the community for a long period. Numerical simulations show that the susceptible unvaccinated females and males and the vaccinated females and males produce periodic oscillations (Figs. 4a, 5a, 6a, b). On the other hand, the phase portrait of the infectious versus susceptible unvaccinated females and infectious versus susceptible unvaccinated males with impulsive vaccination show a decline in the number of susceptible and increase in the number of infected individuals and gradually converge to the endemic equilibrium. According to Theorem 4.1, the disease will be persistent and a positive constant $q$ will exist such that any positive solution $\left(S_{\mathrm{uf}}(t), I_{\mathrm{f}}(t), S_{\mathrm{vf}}(t), S_{\mathrm{um}}(t), I_{\mathrm{m}}(t), S_{\mathrm{vm}}(t)\right)$ of the system Eq. (4) satisfies $I_{\mathrm{f}}(t) \geq q$ and $I_{\mathrm{m}}(t) \geq q$ for $t$ large enough (Figs. 4, 5).

The time series of the susceptible unvaccinated and vaccinated females and males are presented in Fig. 6. Following the application of pulse vaccination, the vaccinated population increases, and then the system returns to the standard SIVS without pulse vaccination model until the next pulse is applied at time $t_{n+1}=t_{n}+5$. 


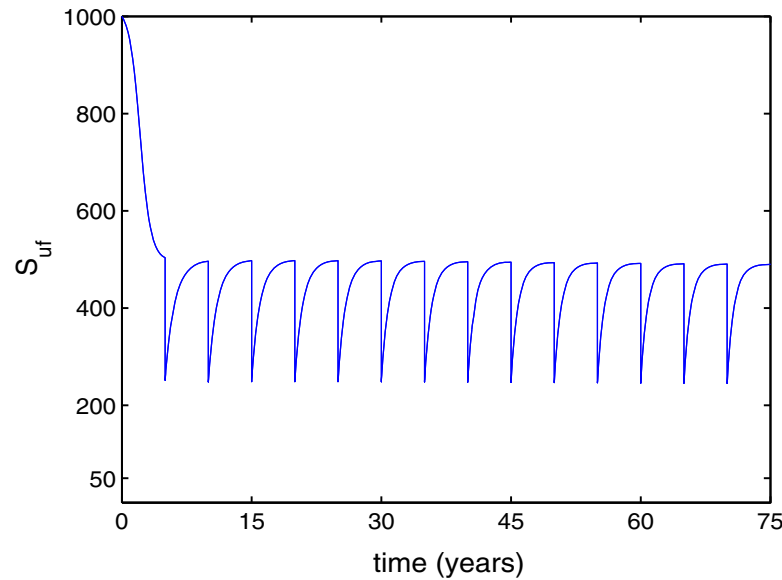

(a) Time series of $S_{u f}$.

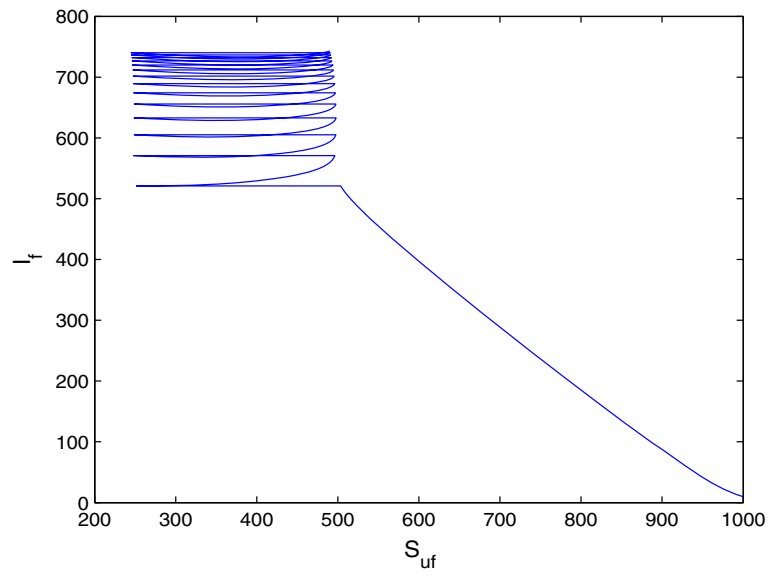

(b) Phase portrait $I_{f}$ vs $S_{u f}$.

Fig. 4 This figure shows the time series of the susceptible unvaccinated females and the phase portrait of $I_{\mathrm{f}}$ versus $S_{\mathrm{uf}}$ with parameter values $T=5, \Pi_{\mathrm{f}}=50, \Pi_{\mathrm{m}}=50, \beta_{\mathrm{m}}=0.001, \beta_{\mathrm{f}}=0.01, \theta_{1}=\theta_{2}=0.5$ and other parameters are as in Table 1

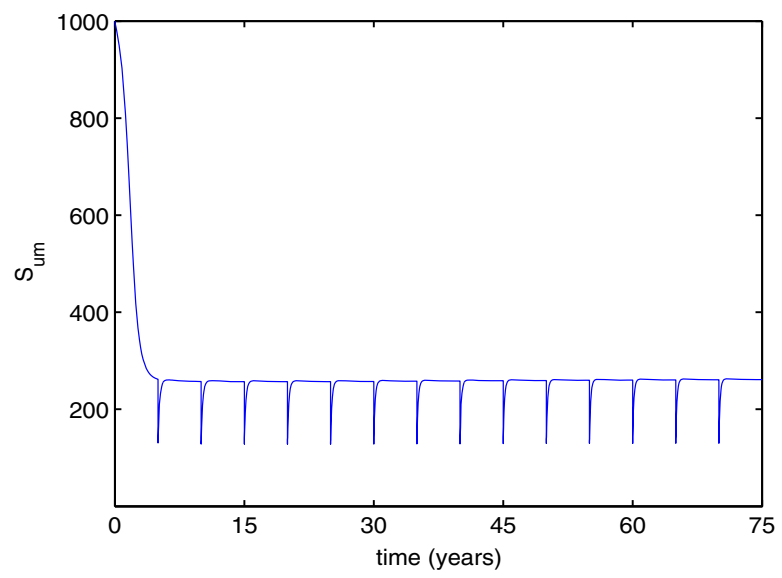

(a) Time series of $S_{u m}$.

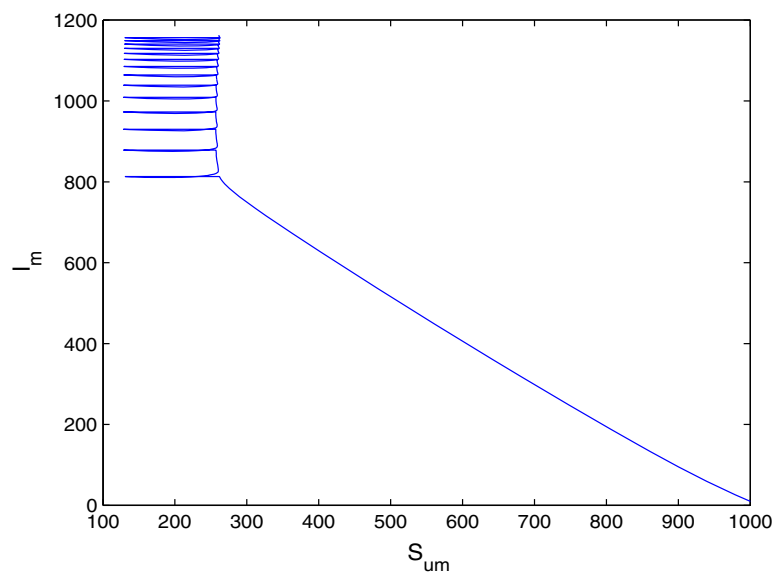

(b) Phase portrait $I_{m}$ vs $S_{u m}$.

Fig. 5 This figure shows the time series of the susceptible unvaccinated males and the phase portrait of $I_{\mathrm{m}}$ versus $S_{\text {um }}$ with parameter values $T=5, \Pi_{\mathrm{f}}=50, \Pi_{\mathrm{m}}=50, \beta_{\mathrm{m}}=0.001, \beta_{\mathrm{f}}=0.01, \theta_{1}=\theta_{2}=0.5$ and other parameters are as in Table 1

\section{Conclusion}

This paper was intended to study the SIVS HPV model with pulse vaccination. The strategy of pulse vaccination (PVS) consists of periodical repetitions of vaccinations in a population that is different from the traditional constant and continuous vaccinations. This method of vaccination is called impulsive because all doses of the vaccine are administered in a very short time as compared to the infectious period of the targeted disease. Using the comparison theorem of impulsive differential equations, the global stability of the disease-free periodic solution is proved. It is found out that the disease-free periodic solution is globally attractive for $R_{0}<1$. This evidence suggests therefore that the disease could die out. It is also obtained that the system is uniformly persistent in the community for $R_{0}>1$. Our results show that a larger pulse vaccination rate given for a larger period of time may result in the eradication of the disease. In order to show the effectiveness of the theoretical findings presented, numerical examples have been given. And the numerical simulations agree with the theoretical results. 


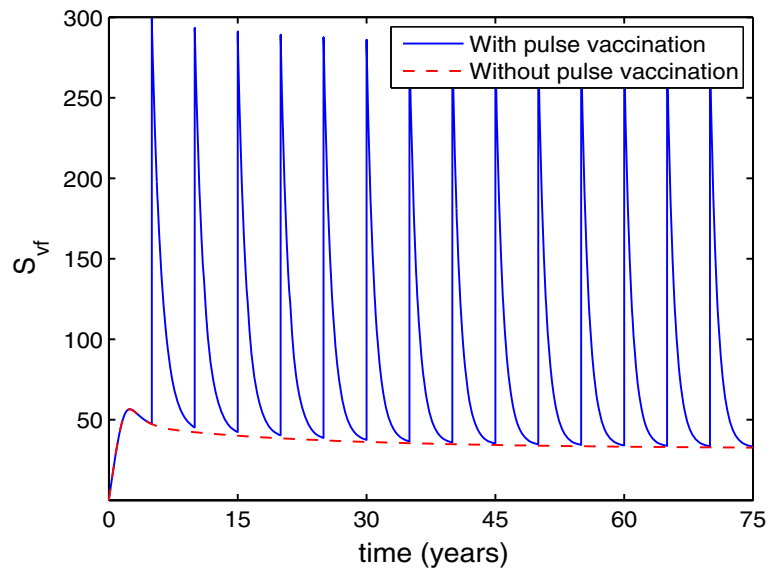

(a) Time series of $S_{v f}$.

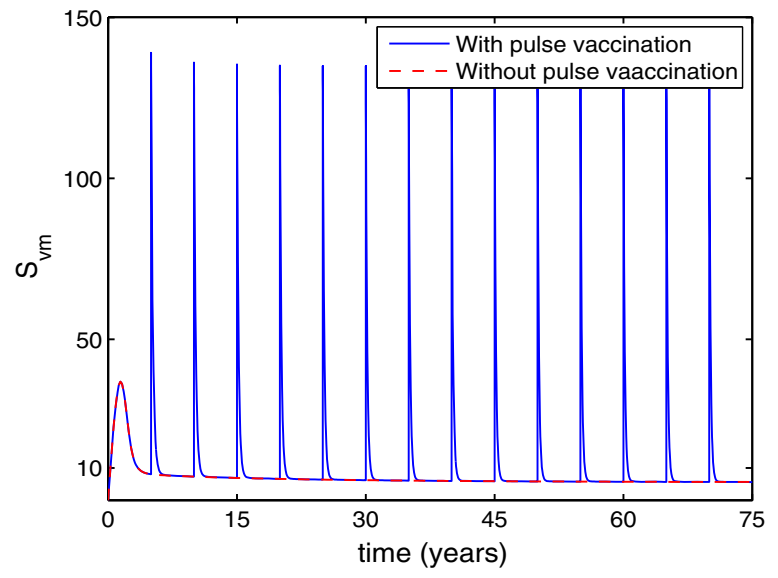

(b) Time series of $S_{v m}$.

Fig. 6 This figure shows the time series of the vaccinated females and males with parameter values $T=5, \Pi_{\mathrm{f}}=50, \Pi_{\mathrm{m}}=50$, $\beta_{\mathrm{m}}=0.001, \beta_{\mathrm{f}}=0.01, \theta_{1}=\theta_{2}=0.5$ and other parameters are as in Table 1

Most previous research on HPV has focused on the implementation of constant and continuous controls $[12,28]$ in reducing the prevalence of the disease. However, some researchers have found out that pulse vaccination is more efficient in defeating certain diseases $[38,39]$. Other authors have also presented some theoretical considerations, practical advantages, and examples of PVS. For example, repeated PVS has been attributed to some successes against poliomyelitis and measles [40,41]. It is found out in this paper that pulse vaccination has a great impact on the reduction of the infectious population, which is in good agreement with the previous researches done.

Finally, several important limitations need to be considered. First, we have considered a model without recovered/or removed class which is against the reality since some infected individuals could get recover permanently. Second, we have considered the infected unvaccinated females and infected vaccinated females as one compartment and call it infected females. In reality, however, the rate of infection of the susceptible unvaccinated females is different from susceptible vaccinated females. The same is true for the male population. It would be interesting to add infected unvaccinated females $I_{\mathrm{uf}}$ and infected vaccinated females $I_{\mathrm{vf}}$ as a separate compartment. Since impulsive differential equations include both discrete and continuous processes, the most significant limitation in the study of stability is that it becomes dif- ficult to obtain a manageable formula for the stroboscopic map as the number of equations increases. To further know the dynamics of the proposed system it is recommended to study the bifurcation analysis.

Acknowledgements The authors are grateful to the editor and the anonymous referees for their constructive comments that have greatly enriched the article.

\section{Compliance with ethical standards}

Conflict of interest The authors declare that they have no conflict of interests.

\section{References}

1. Marcellusi, A.: Impact of HPV vaccination: health gains in the Italian female population. Popul. Health Metrics (2017). https://doi.org/10.1186/s12963-017-0154-0

2. Elbasha, E.H., Dasbach, E.J., Insinga, R.P.: Model for assessing human papillomavirus vaccination strategies. Emerg. Infect. Dis. 13(1), 28-41 (2007). https://doi.org/10. 3201/eid1301.060438

3. World Health Organization, human papillomavirus (hpv) and cervical cancer 2020. https://www.who.int/news-room/fact-sheets/detail/ human-papillomavirus-(hpv)-and-cervical-cancer (2020)

4. Lemos-Paiao, A.P., Silva, C.J., Torres, D.F.: An epidemic model for cholera with optimal control treatment. J. Comput. Appl. Math. 318, 168-180 (2017). https://doi.org/10.1016/ j.cam.2016.11.002

5. Berhe, H.W., Makinde, O.D.: Computational modelling and optimal control of measles epidemic in human population. 
Biosystems 190, 104102 (2020). https://doi.org/10.1016/j. biosystems.2020.104102

6. Subchan, I., Fitria, A.M.: Syafi, An epidemic cholera model with control treatment and intervention. J. Phys. Conf. Ser. 1218, 012046 (2019). https://doi.org/10.1088/1742-6596/ $1218 / 1 / 012046$

7. Berhe, H.W., Makinde, O.D., Theuri, D.M.: Optimal control and cost-effectiveness analysis for dysentery epidemic model. Appl. Math. Inf. Sci. 12, 1183-1195 (2018)

8. Hui, J., Chen, L.: Impulsive vaccination of sir epidemic models with nonlinear incidence rates. Discrete Contin. Dyn. Syst. B 4(3), 595-605 (2004). https://doi.org/10.3934/ dcdsb.2004.4.595

9. Meng, X., Chen, L.: The dynamics of a new sir epidemic model concerning pulse vaccination strategy. Appl. Math. Comput. 197(2), 582-597 (2008). https://doi.org/10.1016/ j.amc.2007.07.083

10. Zhang, T., Teng, Z.: Pulse vaccination delayed seirs epidemic model with saturation incidence. Appl. Math. Model. 32(7), 1403-1416 (2008). https://doi.org/10.1016/j.apm. 2007.06.005

11. Gao, S., Chen, L., Teng, Z.: Impulsive vaccination of an seirs model with time delay and varying total population size. Bull. Math. Biol. 69(2), 731-745 (2006). https://doi. org/10.1007/s11538-006-9149-x

12. Abouelkheir, I., El Kihal, F., Rachik, M., Elmouki, I.: Optimal impulse vaccination approach for an sir control model with short-term immunity. Mathematics 7(5), 420 (2019). https://doi.org/10.3390/math7050420

13. Liu, H., Li, L.: A class age-structured HIV/AIDS model with impulsive drug-treatment strategy. Discrete Dyn. Nat. Soc. 2010, 1-12 (2010). https://doi.org/10.1155/2010/758745

14. Benchohra, M., Henderson, J., Ntouyas, S.: Impulsive Differential Equations and Inclusions. Hindawi Publishing Corporation, London (2006)

15. Bainov, D., Simeonov, P.S.: Impulsive Differential Equations: Periodic Solutions and Applications. Longman Scientific and Technical, London (1993)

16. d'Onofrio, A.: Stability properties of pulse vaccination strategy in seir epidemic model. Math. Biosci. 179(1), 57-72 (2002). https://doi.org/10.1016/s0025-5564(02)00095-0

17. Zhou, Y., Liu, H.: Stability of periodic solutions for an sis model with pulse vaccination. Math. Comput. Model. 38(3-4), 299-308 (2003). https://doi.org/10.1016/ s0895-7177(03)90088-4

18. Abbasi, Z., Zamani, I., Mehra, A.H.A., Shafieirad, M., Ibeas, A.: Optimal control design of impulsive sqeiar epidemic models with application to covid-19. Chaos Solitons Fractals 139, 110054 (2020). https://doi.org/10.1016/j.chaos.2020. 110054

19. Jiao, J., Cai, S., Li, L.: Impulsive vaccination and dispersal on dynamics of an sir epidemic model with restricting infected individuals boarding transports. Physica A 449, 145-159 (2016). https://doi.org/10.1016/j.physa.2015. 10.055

20. Jiao, J., Cai, S., Li, L.: Dynamics of a delayed SEIR epidemic model with pulse vaccination and restricting the infected dispersal. Commun. Math. Biol. Neurosci. (2017). https:// doi.org/10.28919/cmbn/3383

21. Zhou, A., Sattayatham, P., Jiao, J.: Dynamics of an sir epidemic model with stage structure and pulse vacci- nation. Adv. Differ. Equ. (2016). https://doi.org/10.1186/ s13662-016-0853-z

22. Lai, J., Gao, S., Liu, Y., Meng, X.: Impulsive switching epidemic model with benign worm defense and quarantine strategy. Complexity 2020, 1-12 (2020). https://doi.org/10. 1155/2020/3578390

23. Yang, C.-X., Nie, L.-F.: Modelling the use of impulsive vaccination to control rift valley fever virus transmission. Adv. Differ. Equ. (2016). https://doi.org/10.1186/ s13662-016-0835-1

24. Jit, M., Gay, N., Soldan, K., Hong Choi, Y., Edmunds, W.J.: Estimating progression rates for human papillomavirus infection from epidemiological data. Med. Decis. Making 30(1), 84-98 (2009). https://doi.org/10.1177/ 0272989x09336140

25. Lee, S.L., Tameru, A.M.: A mathematical model of human papillomavirus (HPV) in the united states and its impact on cervical cancer. J. Cancer 3, 262-268 (2012). https://doi. org/10.7150/jca.4161

26. Llamazares, M., Smith, R.J.: Evaluating human papillomavirus vaccination programs in canada: Should provincial healthcare pay for voluntary adult vaccination? BMC Public Health (2008). https://doi.org/10.1186/1471-2458-8-114

27. Chanthavilay, P., Reinharz, D., Mayxay, M., Phongsavan, K., Donald, E.M., Moore, L., Lisa, J.W.: The economic evaluation of human papillomavirus vaccination strategies against cervical cancer in women in lao pdr: a mathematical modelling approach. BMC Health Serv. Res. 16(418), 1-10 (2016)

28. Salda'a, F., Korobeinikov, A., Barradas, I.: Optimal control against the human papillomavirus: protection versus eradication of the infection. Abstr. Appl. Anal. 2019, 1-13 (2019). https://doi.org/10.1155/2019/4567825

29. Al-Arydah, M., Malik, T.: An age-structured model of the human papillomavirus dynamics and optimal vaccine control. Int. J. Biomath. 10(06), 1750083 (2017). https://doi. org/10.1142/s1793524517500838

30. Al-arydah, M., Smith, R.: An age-structured model of human papillomavirus vaccination. Math. Comput. Simul. 82(4), 629-652 (2011). https://doi.org/10.1016/j.matcom. 2011.10.006

31. Peng, H.-L., Tam, S., Xu, L., Dahlstrom, K.R., Wu, C.-F., Fu, S., Zhong, C., Chan, W., Sturgis, E.M., Ramondetta, L.E.A.: Age-structured population modeling of hpv-related cervical cancer in Texas and US. Sci. Rep. (2018). https:// doi.org/10.1038/s41598-018-32566-0

32. Lakshmikantham, V., Bainov, D.D., Simeonov, P.S.: Theory of Impulsive Differential Equations. World Scientific, Singapore (1989)

33. Mirko, B.: A comparison theorem of differential equations. Novi Sad J. Math. 40(1), 55-56 (2010)

34. McNabb, A.: Comparison theorems for differential equations. J. Math. Anal. Appl. 119(1-2), 417-428 (1986). https://doi.org/10.1016/0022-247x(86)90163-0

35. Munoz, N., Mendez, F., Posso, H., Molano, M., vandenBrule, A., Ronderos, M., Meijer, C., Munoz, 1.: Incidence, duration, and determinants of cervical human papillomavirus infection in a cohort of Colombian women with normal cytological result. J. Infect. Dis. 190(12): 2077-2087. https://doi.org/10.1086/425907 (2004) 
36. Anic, G.M., Giuliano, A.R.: Genital hpv infection and related lesions in men. Prev. Med. 53, S36-S41 (2011). https://doi.org/10.1016/j.ypmed.2011.08.002

37. Chesson, H.W., Laprise, J.-F., Brisson, M., Markowitz, L.E.: Impact and cost-effectiveness of 3 doses of 9-valent human papillomavirus (hpv) vaccine among us females previously vaccinated with 4-valent hpv vaccine. J. Infect. Dis. 213(11), 1694-1700 (2016). https://doi.org/10.1093/infdis/jiw046

38. Shulgin, B.: Pulse vaccination strategy in the SIR epidemic model. Bull. Math. Biol. 60(6), 1123-1148 (1998). https:// doi.org/10.1016/s0092-8240(98)90005-2

39. Stone, L., Shulgin, B., Agur, Z.: Theoretical examination of the pulse vaccination policy in the SIR epidemic model. Math. Comput. Model. 31(4-5), 207-215 (2000). https:// doi.org/10.1016/s0895-7177(00)00040-6
40. Nokes, D., Swinton, J.: Vaccination in pulses: A strategy for global eradication of measles and polio? Trends Microbiol. 5(1), 14-19 (1997). https://doi.org/10.1016/ s0966-842x(97)81769-6

41. Nokes, D.J., Swinton, J.: The control of childhood viral infections by pulse vaccination. Math. Med. Biol. 12(1), 2953 (1995). https://doi.org/10.1093/imammb/12.1.29

Publisher's Note Springer Nature remains neutral with regard to jurisdictional claims in published maps and institutional affiliations. 